\title{
Cell Dynamics Simulations of Sphere-Forming Diblock Copolymers in 2 Thin Films on Chemically Patterned Substrates
}

\author{
3 Maria Serral, ${ }^{\dagger}$ Marco Pinna, ${ }^{*}+{ }^{+}$Andrei V. Zvelindovsky, ${ }^{\ddagger}$ and Josep Bonet Avalos*, ${ }^{\dagger}$ \\ $4{ }^{\dagger}$ Department d’Enginyeria Química, Universitat Rovira i Virgili, Tarragona, Av. dels Països Catalans, 26, 43007 Tarragona, Spain \\ 5 "School of Mathematics and Physics, College of Science, University of Lincoln, Brayford Pool, Lincoln, Lincolnshire LN6 7TS, U.K.
}

6 S Supporting Information

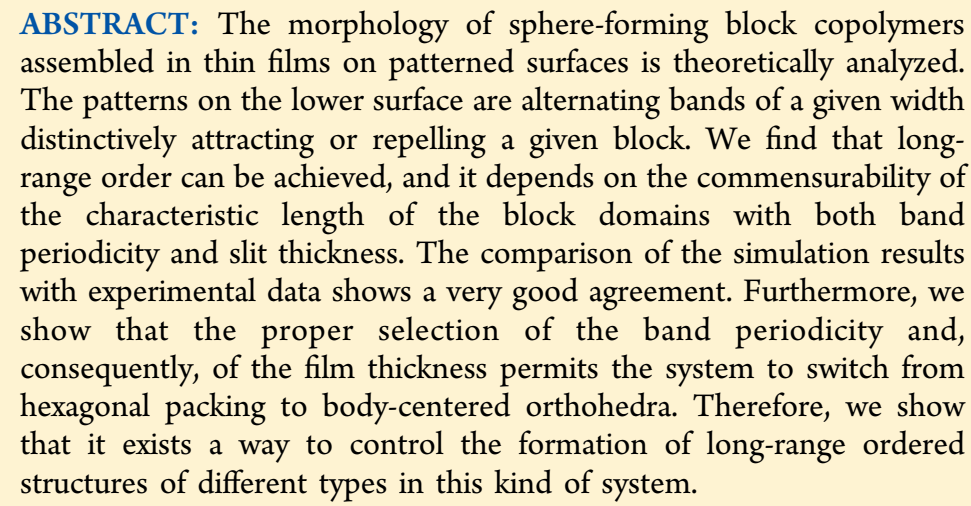
distinctively attracting or repelling a given block. We find that longrange order can be achieved, and it depends on the commensurability of the characteristic length of the block domains with both band periodicity and slit thickness. The comparison of the simulation results with experimental data shows a very good agreement. Furthermore, we show that the proper selection of the band periodicity and, consequently, of the film thickness permits the system to switch from hexagonal packing to body-centered orthohedra. Therefore, we show that it exists a way to control the formation of long-range ordered structures of different types in this kind of system.

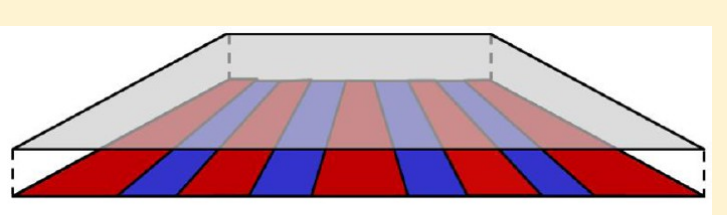

Setup Box

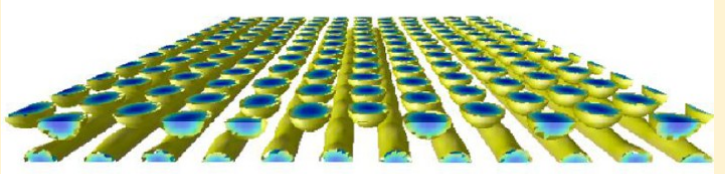

BCP assembly

\section{INTRODUCTION}

20 Supramolecular structures of nanometric size are of great 21 interest due to potential applications in nanotechnology, which 22 range from nanopatterned surfaces, nanocrystals with exotic 23 optoelectronic properties, to functional foods and pharmaco24 logical products. ${ }^{1-3}$ The main technological problem is to 25 achieve the control and reliability of the final product when 26 traditional manufacturing processes cannot be downscaled to 27 the nanometric size. Hence, physical systems that sponta28 neously form supramolecular assemblies under appropriate 29 conditions are so important, as far as they can be externally 30 controlled to produce the desired target structure. ${ }^{4}$ Block 31 copolymers (BCP) are one of these valuable materials because 32 of their ability to self-assemble into different nanostructures. ${ }^{5-9}$ 33 In this article we address the problem of how external effects, 34 like the presence of a chemical mask on a surface, affects the 35 two- and three-dimensional structure of thin films of sphere 36 forming BCP. This question was experimentally analyzed by 37 Park et al., ${ }^{10}$ showing some interesting aspects of the self38 organization of BCP in the presence of a structured wall. Here 39 we apply cell dynamics simulation (CDS) to widen the range of 40 conditions experimentally analyzed and, more importantly, to 41 infer the relationship between the aforementioned external 42 constraints and the particular features of the structures 43 obtained. We find that the surface pattern allows to selectively 44 produce nonbulk types of structures in BCP films.

45 BCP consist of blocks of chemically different monomers 46 covalently bond. The simplest systems are (A-B) diblock 47 copolymers. If monomers $\mathrm{A}$ and $\mathrm{B}$ are highly incompatible, the 48 system can spontaneously segregate into two distinct nano49 domains (microphase separation). The size of such nano- domains is on the range between 10 and $100 \mathrm{~nm}$. Depending 50 on the nature of the monomers, such as their degree of 51 polymerization, interaction between the components, and the 52 relative size of each block, these microphases can be either 53 lamellae, cylinders, or spheres, although other more exotic 54 morphologies, like the gyroid phase, can be observed. ${ }^{11-13} 55$

Morphologies not accessible in the bulk can exist under 56 confinement due to the additional constraints imposed by the 57 surfaces on the system. ${ }^{14}$ Constraints can be spatial frustration, 58 preferential attraction of one of the blocks to the walls with 59 respect to the other, and structures at the interfaces, among 60 others. The confinement geometry can hinder the system in 61 one, two, or three dimensions, ${ }^{15,16}$ as in confinement in thin 62 films, in cylindrical, ${ }^{17-20}$ or spherical pores. ${ }^{21}$

Focusing our attention on thin films, a variety of domain 64 architectures can be obtained influenced by the boundary 65 conditions at both the top and bottom interfaces. ${ }^{22-25}$ BCP can 66 serve to pattern large areas of regular arrays of nanostructures 67 for nanolitography, for the synthesis of highly ordered 68 dispersions of inorganic particles. They can be used for 69 semiconductors, magnetic storage media, photonic crystals, and 70 nanostructured membrane applications, among many 71 others. $^{5-9}$ Almost invariably, microdomains with a long-range 72 order are required for these applications. However, in real 73 systems long-range order is hindered by a rather degenerate 74 free-energy landscape as well as by the slow diffusion of the 75 polymers in the process of domain reorganization after 76

Received: October 22, 2015

Revised: January 4, 2016 
77 quench. $^{26,27}$ It has been shown that perfectly ordered arrays of 78 domains in thin films of BCP are not expected even at 79 equilibrium, since in a nearly $2-\mathrm{D}$ system the translational order 80 is at best quasi-long-range due to a finite concentration of 81 thermally activated defects. ${ }^{28,29}$ Moreover, hexatic phases with 82 no long-range translational order appear at temperatures close 83 to the melting of the structure into a disordered phase of 84 domains. $^{27} \mathrm{~A}$ review on the importance of the spontaneous 85 defects as well as the kinetically arrested imperfections in direct 86 self-assembly of BCP has been recently published by $\mathrm{Li}$ and 87 Müller. $^{30}$

88 Various techniques have been proposed to tailor the 89 arrangements of nanoscale domains, or to accelerate the slow 90 dynamics of the ordering near equilibrium, to produce the 91 aforementioned well-controlled long-range order. One can find 92 the modifications in the supporting substrates, such as 93 topographical or chemical patterning and the application of 94 external fields, like shear or electrical fields. ${ }^{1,31}$ For symmetric 95 (lamellae forming) BCP the commensurability between the 96 periodicity of the pattern substrate and the natural length scale 97 of the BCP was found to play an indispensable role to obtain a 98 particular lamellar morphology in thin films. ${ }^{32}$ Subsequently, 99 regular almost defect-free linear conformations in the nanoscale 100 could be fabricated. ${ }^{33-35}$ As far as the asymmetric block 101 copolymers are concerned, dense arrays of nanocylinders with 102 controlled orientation and enhanced uniformity are obtained 103 using hexagonal arrays of dots chemically patterned on the 104 supporting substrate. ${ }^{36-39}$ Square arrays of vertical cylinders, 105 instead of the hexagonal array typically found in bulk, can also 106 be produced using a chemically patterned surface with a square 107 array of spots. ${ }^{40}$

108 With regards to the sphere-forming BCP in thin films, long109 ranged order structures are achieved on topographically 110 modified surfaces forming wells and mesas, which yield 111 crystal-like structures. ${ }^{41}$ On the other hand, when assembling 112 spheres in V-shaped grooves, well-ordered domains are 113 obtained with a face-centered cube (FCC) close-packing 114 structure, with the (111) planes of the array parallel to the 115 groove walls. $^{42}$ Additionally, hexagonally arranged posts or 116 semidense hole patterns ${ }^{43,44}$ can be used to form arrays of 117 spherical domains whose periodicity is larger than the one of 118 the substrate, a phenomenon referred to as density multi119 plication. The orientation and periodicity of the resulting array 120 of spherical microdomains are found to be governed by the 121 commensurability between the $\mathrm{BCP}$ microphase characteristic 122 length and the template periodicity. Alternatively, well-aligned 123 spherical domains built using a chemically striped patterned 124 surface, also with a periodicity commensurate with the BCP 125 natural periodicity, were reported. ${ }^{10}$ Furthermore, the film 126 thickness is also a key parameter to draw long-range order of 127 spherical BCP domains, as can be inferred from the transitions 128 between the typical 3-D body-centered cube (BCC) arrange129 ment of spheres found in the bulk and the 2-D hexagonal 130 (HEX) arrangement encountered under confinement. ${ }^{26,45}$

131 Recent efforts have been directed to increase the accuracy of 132 the control over the shapes and dimensions of the equilibrium 133 morphologies. ${ }^{46,47}$ The goal is to improve defect-free long134 range order arrangements, aiming at obtaining high density 135 multiplication, together with pattern quality rectification and 136 fabrication of new complex structures never found in the 137 bulk. ${ }^{48-52}$ Müller et al. ${ }^{4}$ recently introduced a new procedure to 138 drive the self-assembly toward desired geometries through a 139 process-directed mechanism in which the kinetics of structure formation is externally led to achieve the sought long-life 140 metastable state.

From the theoretical viewpoint, two main approaches have 142 been widely used to describe the microphase separation of 143 BCP. On the one hand, the self-consistent field theory (SCFT) 144 deals with coarse-grained models of the BCP in which bonded 145 interactions are described as flexible Gaussian chains, while 146 nonbonded interactions are introduced through the mean local 147 densities. Many calculations in 2 and 3 dimensions for relatively 148 small systems have been carried out aiming at understanding 149 the formation of BCP structures. ${ }^{53-57}$ In this line, simulations 150 of confined systems, with chemically activated or topo- 151 graphically modified substrates, were performed by several 152 authors. ${ }^{36,58-61}$ Although the method permits to keep some 153 molecular details of the chain, SCFT is still a computationally 154 expensive method when large systems are to be studied. 155 Particularly, the dynamic processes involving domain restructu- 156 ration are very long as compared with the molecular scales of 157 chain reorganization, whose specific consideration is required in 158 a dynamic implementation of the SCFT. Other particulate 159 models, also based on coarse-grained descriptions of the BCP 160 molecules, have also been constructed and used in several 161 studies. Early lattice Monte Carlo simulations of simple models 162 were used to analyze BCP in confined geometries, including 163 also patterned surfaces. ${ }^{62,63}$ More recently, it was introduced 164 the so-called theoretically informed coarse-grained simulations 165 (TICG), ${ }^{64,65}$ which permit the study of the BCP dynamics 166 through dynamic Monte Carlo methods, for instance. Although 167 coarse-grained, this method allows for the study of multi- 168 component systems, suspended particles, can reproduce 169 thermal fluctuations (i.e., spontaneously generate thermal 170 defects), etc., and still be computationally efficient. Together 171 with the SCFT, TICG approximately described the conforma- 172 tional changes of the BCP chains in the vicinity of a interface. 173 However, the explicit considerations of coarse-grained chain 174 conformations limits the affordability of simulations of large 175 systems or long-time ranges.

On the other hand, Ohta and Kawasaki ${ }^{66}$ developed a free- 177 energy functional corresponding directly to the monomer 178 concentration fields, with no explicit reference to microscopic 179 details, except in the definition of the coefficients. Its final form 180 resembles a Ginzburg-Landau functional supplemented with a 181 long-range contribution that accounts for the chain con- 182 nectivity. From the free-energy functional a diffusive dynamics 183 can be constructed in the spirit of the Cahn-Hilliard-Cook 184 (CHC) equation ${ }^{67}$ for systems with a conserved order 185 parameter. This coarse-grained level of description allows to 186 observe the microphase separation phenomena in systems 187 whose dimensions are comparable with the ones experimentally 188 addressed. This method is customarily referred to as cell 189 dynamics simulation. ${ }^{68,69}$ In the framework of this method the 190 dynamics and structure of BCP on chemically patterned 191 surfaces have also been studied for cylinder and lamellae 192 forming BCP. ${ }^{70-75}$

However, the theoretical analysis of the self-assembly of 194 spheres on a chemically patterned surface has not been 195 addressed yet. In this article we explore the usefulness of a very 196 simple stripped pattern in guiding the final structure of 197 asymmetric sphere forming BCP in contrast to more 198 sophisticated designs like arrays of spots or other graph- 199 oepitaxial techniques. The numerical approach allows us to 200 infer the effect of the commensurability between the pattern 201 periodicity, as well as the slit thickness, and the natural spacing 202 
203 of the formed structures. The analysis permits us to identify the 204 optimal conditions for defect-free assemblies of several layers of 205 spheres, with different unit cell. The study reveals some keys for 206 a better structure control in the manufacturing at the nanoscale. 207 The paper is organized as follows. In section 2, we introduce 208 the theoretical background of the CDS and its application to a 209 system implemented on a lattice. Special emphasis is made in 210 deriving the appropriate free-energy functional for the lattice. In 211 section 3, we analyze the theoretical results and compare them 212 with the available experimental data. Predictions for a wider 213 range of conditions are also discussed. Finally, section 4 is 214 devoted to review the main conclusions that can be drawn from 215 our work.

\section{MODEL}

216 Cell Dynamics Simulation. The cell dynamics simulation 217 method was introduced to model interface dynamics in phase218 separating systems. Theoretically, CDS is based on the Cahn219 Hilliard-Cook equation, which is used as the governing 220 differential equation for the time evolution of a conserved order 221 parameter $\Psi(\mathbf{r}, t){ }^{68,76}$ The $\mathrm{CHC}$ equation can be derived from 222 the balance equation for the order parameter together with the 223 linear relationship between the order parameter flux $\mathbf{J}(\mathbf{r}, t)$ and 224 the local chemical potential, ${ }^{77}$ eqs 1 and 2, respectively. That is

${ }_{225} \quad \frac{\partial \Psi(\mathbf{r}, t)}{\partial t}=-\nabla \cdot \mathbf{J}(\mathbf{r}, t)$

$226 \mathbf{J}(\mathbf{r}, t)=-M \nabla \mu(\mathbf{r}, t)$

227 The chemical potential can be expressed as a functional 228 derivative of the free energy functional

229

$$
\mu(\mathbf{r}, t)=\frac{\delta F[\Psi]}{\delta \Psi(\mathbf{r}, t)}
$$

230 Hence, the $\mathrm{CHC}$ equation is obtained as

$$
231 \quad \frac{\partial \Psi}{\partial t}=M \nabla^{2}\left(\frac{\delta F[\Psi]}{\delta \Psi}\right)
$$

232 where $M$ is a phenomenological mobility constant taken as $M=$ 2331 . This choice sets the time scale for the diffusive processes (the 234 dimensionless time is $t M / a_{0}^{2}$, where the lattice cell spacing $a_{0}$ is 235 taken as the unit of length). The dynamics of eq 4 is consistent 236 with the mean-field theory and would correspond to a situation 237 with negligible thermal fluctuations, namely, BCP of large 238 molecular weight ${ }^{78,79}$ (see the Supporting Information for a 239 discussion on the description of thermal noise in CDS; also see 240 Zvelindovsky and Zatovsky, ${ }^{80}$ van Vlimmeren et al., ${ }^{81}$ Knoll et 241 al., ${ }^{82}$ and Horvat et al. ${ }^{83}$ ).

242 In CDS, the commonly used expression for the order 243 parameter $\Psi(\mathbf{r}, t)$ in terms of the $A$ and $B$ comonomer volume 244 fractions, $\phi_{A}$ and $\phi_{B}$, respectively, is ${ }^{68,69}$

$245 \quad \Psi(\mathbf{r}, t)=\phi_{A}(\mathbf{r}, t)-\phi_{B}(\mathbf{r}, t)+(1-2 f)$

246 where $f=N_{A} /\left(N_{A}+N_{B}\right)$ stands for the number fraction of $A$ 247 monomers per chain. The order parameter is then the 248 difference between the local volume fraction of both 249 monomers, plus a constant such that the integral of $\Psi(\mathbf{r}, t)$ 250 over the entire volume of the system is 0 by construction. For 251 symmetric copolymers, $f=1 / 2$, the constant vanishes.

252 The necessary condition of equilibrium is given by

$$
\left.\frac{\delta F[\Psi]}{\delta \Psi}\right|_{\mathrm{eq}}=0
$$

with a positive second functional derivative. Equation 6 is not a 254 sufficient condition because the system can be trapped into 255 local metastable minima during its time evolution according to 256 eq 4.

The free energy $F[\Psi]$ (in reduced units such that $k T=1$ ) is 258 given by ${ }^{66,84}$

$$
\begin{aligned}
& F[\Psi(\mathbf{r})]=\int \mathrm{d} \mathbf{r}\left\{\left[H(\Psi)+\frac{D}{2}|\nabla \Psi|^{2}\right]+\frac{B}{2} \int \mathrm{d} \mathbf{r}^{\prime}\right. \\
& \left.\times G\left(\mathbf{r}-\mathbf{r}^{\prime}\right) \Psi(\mathbf{r}) \Psi\left(\mathbf{r}^{\prime}\right)-\sum_{i} s_{i}(\mathbf{r}) \Psi(\mathbf{r})\right\}
\end{aligned}
$$

The first term, $H(\Psi)$, contains the local contributions to the 261 free energy, expressed as an expansion in terms of the order 262 parameter, in the spirit of the Ginzburg-Landau theory. ${ }^{66,85} 263$ The expression used in the current analysis is given by ${ }^{68,86} \quad 264$

$$
H(\Psi)=\left[-\frac{\tau}{2}+\frac{A}{2}(1-2 f)^{2}\right] \Psi^{2}+\frac{v}{3}(1-2 f) \Psi^{3}+\frac{u}{4} \Psi^{4}
$$

Here, $A, v$, and $u$ are phenomenological constants derived by 266 Leibler $^{85}$ using the random phase approximation, and $\tau$ is 267 proportional to the Flory-Huggins parameter $\chi$, which 268 inversely depends on the temperature T. According to Ohta 269 and Kawasaki, ${ }^{66,85}$ the parameter

$$
\tau^{\prime} \equiv-\tau+A(1-2 f)^{2}
$$

can be expressed in terms of physical parameters as

$$
\tau^{\prime}=-\frac{1}{2 N}\left(N \chi-\frac{\tilde{s}(f)}{4 f^{2}(1-f)^{2}}\right)
$$

where $\tilde{s}(f)$ is an empirical fitting function whose value is of 274 order of $1 .{ }^{66}$

The surface term, $D|\nabla \Psi|^{2} / 2$, accounts for the free energy 276 necessary to create an interface between $A$ and $B$. The 277 coefficient $D$ is a positive constant that scales as $D \sim b^{2}$, where 278 $b$ is the Kuhn segment length of the polymer. The expression 279 for $D$ reads ${ }^{85}$

280

$$
D=\frac{b^{2}}{48 f(1-f)}
$$

Additionally, chain connectivity introduces a long-range term in 282 eq 7 , with the coefficient $B$ that scales as $N^{-2}$, and reads ${ }^{66,68,85} 283$

$$
B=\frac{9}{(2 N b f(1-f))^{2}}
$$

where $N=N_{A}+N_{B}$ is the total degree of polymerization. This 285 term carries a chain-length dependence. Notice that the Green 286 function $G\left(\mathbf{r}-\mathbf{r}^{\prime}\right)$ satisfies $\nabla^{2} G\left(\mathbf{r}-\mathbf{r}^{\prime}\right)=-\delta(\mathbf{r}-\mathbf{r} 287$ '). ${ }^{66,68,85,86}$ It should be mentioned here that this particular 288 form of the Green function was derived for bulk systems. The 289 presence of hard walls is expected to influence the functional 290 form of the Green function due to the reduction of the number 291 of chain conformations when polymers are near an 292 impenetrable surface, as follows from its derivation. ${ }^{66,85} 293$ However, this effect qualitatively does not introduce significant 294 deviations in the predictions, as can be seen from the 295 
296 comparison with experimental data of related systems later on. 297 For convenience, we define the dimensionless parameters $\tilde{D}=$ $298 D / a_{0}{ }^{2}$ and $\tilde{B}=B a_{0}{ }^{2}$ to be used in the simulations. For 299 simplicity, we write $D$ and $B$ instead of $\tilde{D}$ and $\tilde{B}$ from now on. 300 The parameters in eqs $7-9$ are considered as phenomeno301 logical constants.

302 The last term in eq 7, where $i$ denotes either $A$ or $B$ 303 component, takes into account the interaction of the block 304 copolymer with the confining hard walls. In the present case, 305 the walls model surfaces presenting a chemically activated mask. 306 Hence, $s_{i}(x, y, z)$ is defined as ${ }^{87,88}$

307

$$
s_{i}(x, y, z)=h_{i} \phi_{i} \tilde{\delta}_{x} \delta_{z, 1}
$$

308

$$
\tilde{\delta}_{x} \equiv \begin{cases}-1, & 2 n w \leq x<(2 n+1) w \\ 1, & (2 n+1) w \leq x<2(n+1) w\end{cases}
$$

309 where $n$ is an integer and $h_{i}$ is the strength of the interaction 310 between the walls and the respective $i$ segments. Moreover, $w$ is 311 the width of an individual stripe, and $\delta_{a b}$ is the Kronecker delta. 312 The pattern on the lower surface thus corresponds to long 313 stripes parallel to the $y$-direction. Notice that $\phi_{i}$ can be 314 expressed in terms of the order parameter $\Psi$, i.e., $\phi_{A}=f+\Psi / 2$ 315 and $\phi_{B}=1-f-\Psi / 2$.

316 Numerical Cell Dynamics Simulation. The numerical 317 scheme introduced to implement the dynamic model described 318 so far is based on the definition of the fields in a cubic lattice, 319 together with an appropriate discretization of the Laplacian in 320 eq $4 .{ }^{89}$ We propose

321

$$
\begin{aligned}
& \Psi(i, j, k ; t+1)=\Psi(i, j, k ; t)-\{\langle\langle\Gamma(i, j, k ; t)\rangle\rangle \\
& -\Gamma(i, j, k ; t)+B \Psi(i, j, k ; t)\}
\end{aligned}
$$

322 Here $(i, j, k)$ stands for the set of indices of a given grid point on 323 the lattice, and $\langle\langle\Gamma\rangle\rangle-\Gamma$ stands for a discrete Laplacian 324 defined by ${ }^{68,90}$

325

$$
\langle\langle\Psi\rangle\rangle=\frac{6}{80} \sum_{N N} \Psi+\frac{3}{80} \sum_{N N N} \Psi+\frac{1}{80} \sum_{N N N N} \Psi
$$

326 where $N N$ denotes nearest neighbors, $N N N$ next-nearest 327 neighbors, and NNNN next-next-nearest neighbors of a point $328(i, j, k)$.

329 The function $\Gamma$ is defined as

$$
\begin{aligned}
& \Gamma(i, j, k ; t)=g(\Psi(i, j, k ; t))-\Psi(i, j, k ; t) \\
& \quad+D[\langle\langle\Psi(i, j, k ; t)\rangle\rangle-\Psi(i, j, k ; t)]-s_{i}(z)
\end{aligned}
$$

331 Finally, the so-called map function $g(\Psi(i, j, k ; t))$ is directly 332 related to the functional derivative of $H$ given in eq 8 . For our 333 particular system it reads ${ }^{84,91}$

$$
\begin{aligned}
& g(\Psi(i, j, k ; t))=\left[1+\tau-A(1-2 f)^{2}\right] \Psi \\
& \quad-v(1-2 f) \Psi^{2}-u \Psi^{3}
\end{aligned}
$$

334

Free Energy Functional on the Lattice. The transition 336 from the continuous space to the lattice introduces necessary 337 prescriptions for the evaluation of the Laplacian and the 338 nonlocal contribution appearing in the definition of the free 339 energy functional, defined in continuous space in eq 7. As a 340 consequence, we cannot construct the corresponding free 341 energy functional model on the lattice by the direct 342 discretization of the right-hand side of eq 7 , expecting that 343 this model has the required properties for the discrete field, namely, that the dynamics implemented in eq 15 progresses 344 monotonously lowering the value of the free energy functional 345 to its minimum at equilibrium. The discrete counterpart of the 346 functional derivative of the free energy functional should be 347 compatible with the second term on the right-hand side of eq 348 15 , after the discrete Laplacian is taken, according to eqs 3 and 349 4.

350

Let $F[\Psi(i, j, k)]$ be the free-energy functional of the field 351 $\Psi(i, j, k)$ defined on the lattice. The counterpart of the 352 functional differentiation on the lattice at a given point $(i, j, k) 353$ is simply the partial derivative with respect to the field at the 354 given point. Hence, the chemical potential in the lattice reads 355

$$
\mu(i, j, k)=\frac{\partial F}{\partial \Psi(i, j, k)}
$$

Accordingly, the dynamic equation (15) can be written as

$$
\Psi(i, j, k ; t+1)=\Psi(i, j, k ; t)+\langle\langle\mu(i, j, k)\rangle\rangle-\mu(i, j, k)
$$

Therefore, the key point is to provide a discrete free energy 359 whose functional derivative on the lattice, eq 19, gives a 360 chemical potential field such that eq 20 is identical to eq 15 . We 361 thus propose

$$
\begin{gathered}
F[\Psi(i, j, k)]=\sum_{i, j, k}\left(H[\Psi]-\frac{D}{2} \Psi[\langle\langle\Psi\rangle\rangle-\Psi]\right. \\
\left.+\frac{B}{2} \mu^{(l r)} \Psi+s_{A}\left[\frac{\Psi}{2}+f\right]\right)
\end{gathered}
$$

where both $\Psi$ and $\mu^{(l r)}$ on the right-hand side of eq 21 are 364 calculated at the grid point $(i, j, k)$. Furthermore, $\mu^{(l r)}$ is the 365 solution of the algebraic set of equations defined from the 366 relation

$$
\left\langle\left\langle\mu^{(l r)}(i, j, k)\right\rangle\right\rangle-\mu^{(l r)}(i, j, k)=-\Psi_{i, j, k}
$$

which corresponds to the discrete Laplacian of the long-range 369 term equated to the thermodynamic force used on eq 15, 370 according to eq 20. One can iteratively solve the set of eqs 22371 and substitute the field as well as the resulting $\mu^{(l r)}$ in eq 21 to 372 obtain the numerical value of the free energy functional for a 373 given field $\Psi$ defined in the lattice.

374

The obtained free-energy functional on the lattice is thus 375 compatible with the implemented dynamic equation and 376 therefore has the desired properties. The value of the free 377 energy at the final state of the simulation is be used to compare 378 the relative stability of the obtained structures.

379

\section{RESULTS AND DISCUSSION}

Model System. In this study we consider a sphere-forming 380 diblock copolymer melt. The CDS parameters are $f=0.4, u=381$ $0.38, v=2.3, B=0.01, D=0.5, A=1.5$, and $\tau=0.2$, which are 382 known to produce spherical domains. ${ }^{92,93}$ The morphological 383 characteristics of such spherical domains are the spacing 384 between spheres $d \simeq 11$ and their average diameter $\phi \simeq 7.8$ in 385 units of lattice spacings $a_{0}$ (see Figure 1). These parameters are $386 \mathrm{fl}$ taken from Pinna et al. ${ }^{93}$ and correspond to the morphology of 387 a monolayer of spheres formed in a slit with homogeneous 388 surfaces selectively attracting the minority A-block.

Our system is BCP confined in a slit of hard walls. The lower 390 surface is decorated with a mask of stripes with different affinity 391 to the blocks of the BCP, i.e., different values of $s_{i}$ in eq 7. In 392 particular, we have chosen $h_{A}=0.1$ and $h_{B}=-0.1$ according to 393 


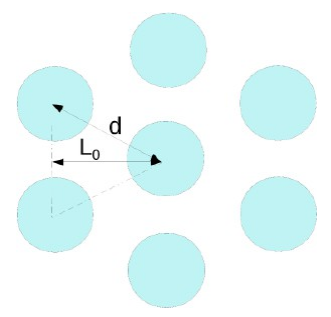

Figure 1. Sphere-froming BCP in a planar hexagonal cell with the characteristic dimensions. The natural lateral period for the hexagonal forming system ${ }^{93}$ is $L_{0} \simeq 9.5\left(L_{0}=\sqrt{3} / 2 d\right)$.

394 eqs 13 and 14. The upper surface of the slit is neutral $\left(s_{i}=0\right.$ for 395 all blocks $i$ ). These solid walls are located at the position $z=1$ 396 for the bottom surface and $z=L_{z}$ for the top surface, as shown 397 in Figure 2a. The pattern on the bottom surface corresponds to

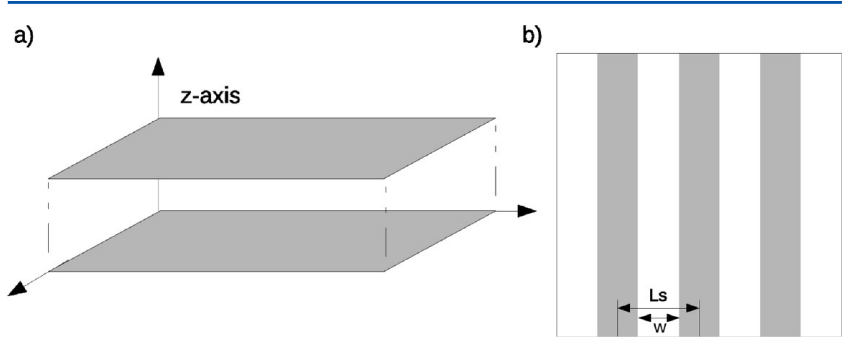

Figure 2. (a) Setup and (b) mask.

398 a set of alternating parallel stripes along the $y$-direction, whose 399 width is $w=L_{S} / 2$, as depicted in Figure $2 \mathrm{~b}$. Thus, the distance 400 between equal stripes (or band periodicity) is $L_{S}$. We have 401 verified that the morphology of the patterns observed is rather 402 insensitive to the attraction energy $h$ if the latter is kept within 403 the range from 0.1 to 0.4 . Hence, for convenience, we have 404 chosen $h=0.1$ in all the simulations. Periodic boundary 405 conditions (PBC) are set for the $x$ - and $y$-directions. The 406 boundary condition of zero flux of $\Psi$ at the hard surfaces 407 implies that the gradient of the chemical potential eq 3 vanishes 408 at the interfaces. ${ }^{87}$ Numerically, such a condition is fulfilled by 409 extending the field inside the wall and demanding $\Psi(x, y, z=0)$ $410=\Psi(x, y, z=1)$ and $\Psi\left(x, y, z=L_{z}+1\right)=\Psi\left(x, y, z=L_{z}\right)$, where $z=$ 4110 and $z=L_{z}+1$ are the coordinates of the extended layers. ${ }^{88}$ 412 We have considered systems of lateral size $L_{x}=L_{y}=120 \simeq$ $41311 d$ with different thicknesses $L_{z}$ ranging from 6 to 56 grid 414 spacings (from $0.5 d$ to $5 d$, approximately). In every case, three 415 different band periodicities of $L_{S}=8,10$, and 12 are studied. 416 These values guarantee that $L_{S}$ is commensurate with $L_{x}$, so 417 that $L_{x} / L_{S}$ is an integer, to comply with the periodic boundary 418 conditions. In the experimental work of Park et al., ${ }^{10}$ the 419 authors have chosen a band periodicity $L_{S} \simeq L_{0}$, where $L_{0}$ is 420 defined in Figure 1 , with $L_{0}=\sqrt{3} / 2 d \simeq 0.86 d$, which 421 corresponds approximately to 9.5 lattice spacings. Variations $422 L_{S}<L_{0}$ and $L_{S}>L_{0}$ were also studied in this last reference. Our 423 choice of the values of $L_{S}$ in the simulation aims at reproducing 424 these experimental situations.

425 The equilibrium structures have been computationally 426 determined by the dynamic evolution of the CDS. The 427 simulation starts from an homogeneous state on which we have 428 superimposed a random perturbation to the order parameter 429 field $\Psi= \pm 0.5$ at every grid point. The deterministic dynamics 430 given in eq 15 progresses until a final state, where no variation 431 in the order parameter field is observed. In general, the evolution leads to spherical domains characterized by values of 432 the field $\Psi \simeq \Psi^{-}$, where $\Psi^{-}$is the negative minimum of $H$ in 433 eq 8. This dynamics corresponds to a quench of the system at a 434 given temperature below the critical temperature, which in our 435 case is $\tau^{\prime}=-0.14$ (for a qualitative analysis of the system, see 436 the Supporting Information). The progress of the quench 437 dynamics starts with a fast process, dominated by spinodal 438 decomposition, in which the domains grow from the local 439 initial inhomogeneities. Then, it follows a much slower process 440 of rearrangements of the domains in space and size. While the 441 former process takes between 1000 and 10000 time steps, the 442 latter takes of the order of $10^{7}$ time steps or longer.

443

The approach to equilibrium can be monitored through the 444 free energy of the system given by eq 21 . However, we cannot 445 guarantee that the final structures, even after our long 446 simulation runs, correspond to true equilibrium arrangements, 447 since they could also be metastable states caused by local free- 448 energy minima. To overcome metastable states, we have added 449 a random noise ${ }^{86,94}$ to the evolution equation, eq 15 , according 450 to

$$
\begin{aligned}
& \Psi(i, j, k ; t+1)=\Psi(i, j, k ; t)-\{\langle\langle\Gamma(i, j, k ; t)\rangle\rangle \\
& -\Gamma(i, j, k ; t)+B \Psi(i, j, k ; t)-\zeta\}
\end{aligned}
$$

where $\zeta$ is a white noise process with a given amplitude. As we 453 have discussed in the previous section, the fluctuation- 454 dissipation theorem does not apply here since this term is a 455 computational artifact and is not intended to reproduce thermal 456 noise. We recall that the physical dynamics is deterministic with 457 the temperature embedded in the parameter $\tau^{\prime}$, which is the 458 control parameter of the phase separation. ${ }^{79}$ Hence, there is no 459 inherent physical temperature scale in the amplitude of $\zeta$ as we 460 use it along this article. In all cases, the final configuration is 461 obtained after setting $\zeta=0$ for at least 100000 time steps 462 before the end.

463

We employ a second dynamic procedure based on annealing. 464 As before, the initial state is a homogeneous field with 465 analogous superimposed random inhomogeneities. Here, 466 instead, the value of $\tau^{\prime}$ is linearly lowered with time. We have 467 chosen the system to decrease from $\tau^{\prime}=0$ to the final $\tau^{\prime}=468$ -0.14 in a given lapse of the order of the total time. When 469 performing the annealing, the free energy does not significantly 470 change until a threshold, which varies with the system, is 471 reached. Then, the free energy drops near the final equilibrium 472 value. Noise is also added to the dynamic evolution and 473 switched off during the last 100000 time steps.

474

The initial stages of two representative free energy 475 trajectories for quenching as well as annealing without added 476 noise are shown in Figure 3. In this example the sudden change $477 \mathrm{f3}$ of slope in the annealing occurs at around $t \simeq 20000,478$ corresponding to $\tau^{\prime} \simeq-0.11$. The dynamic routes to 479 equilibrium are very different, depending on whether 480 quenching or annealing is chosen, meaning that the system 481 evolves through different intermediate structures depending on 482 the route. In Figure 4 we show a sequence of intermediate $483 \mathrm{f} 4$ structures from the same initial state and leading to the same 484 final state, for both quenching and annealing. The final states 485 are compared with regard to their free energy to elucidate 486 which is more stable. In the figures, only A-rich regions are 487 shown while the empty space correspond to B-rich regions. 488

Without the addition of the noise, annealing would invariably 489 produce metastable cylinders even close to the final temper- 490 ature $\tau^{\prime}=-0.14$. The noise, however, moves the system toward 491 


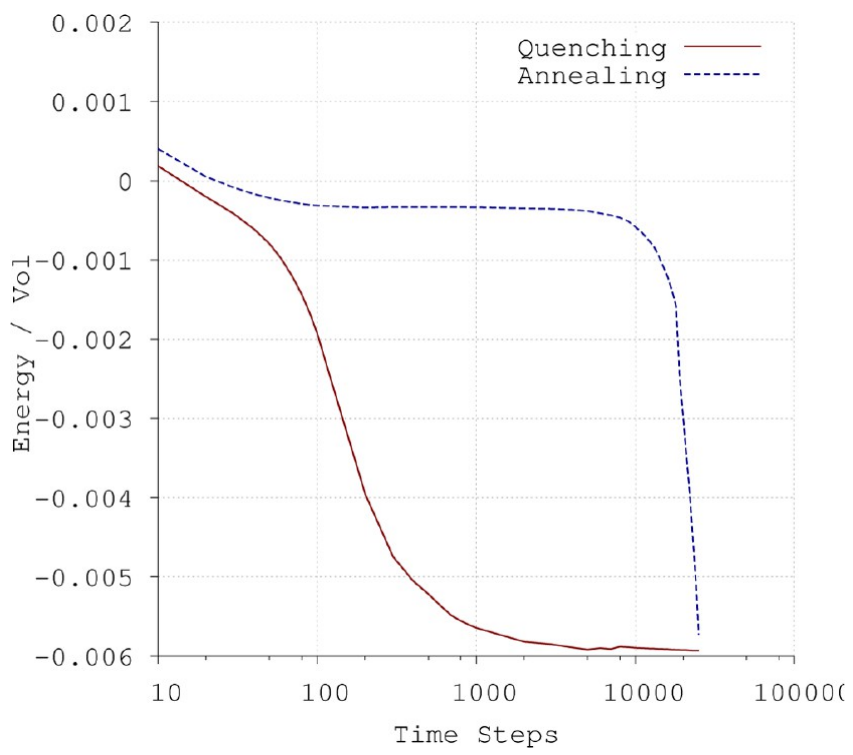

Figure 3. Initial steps of the free energy evolution for the quenching and the annealing processes without added noise.

492 lower energy configurations consisting of ordered arrangements 493 of spheres. Annealing is particularly useful for frustrated 494 situations where quenching often leads to a final state 495 consisting of a disordered array of spheres. The evaluation of 496 the free energy of the system permits us to discriminate the 497 more stable configuration when the two routes produce 498 different results for the same system.

499 Slits $L_{z} \leq \boldsymbol{d}$. Let us consider narrow slits whose thickness is 500 of the order of or smaller than the BCP characteristic domain 501 separation $d$, which is proportional to the polymer chain 502 length, ${ }^{66} d \sim N^{2 / 3}$ (see also the Supporting Information). The 503 analysis of this particular case permits us to check the validity of 504 the model for the study of BCP systems under confinement. 505 Park et al. ${ }^{10}$ experimentally studied $\mathrm{P}(\mathrm{S}-b-\mathrm{MMA})$ on 506 chemically patterned surfaces giving spherical domains with $\phi$ $\simeq 26.5 \mathrm{~nm}$ and $d \simeq 65.2 \mathrm{~nm}$. Their experimental systems 507 consist of a slit with different thicknesses and an area of $30 \times 508$ $400 \mu \mathrm{m}^{2}$. On the bottom surface the system presents a striped 509 (line-and-space) pattern, with a periodicity (band periodicity) 510 ranging from $L_{S}=50.0$ to $60.0 \mathrm{~nm}$. The systems with $L_{z}=25511$ $\mathrm{nm}$, which correspond to a slit thickness significantly smaller 512 than $d$, produce scattered domains with no order and a wetting 513 layer for each block on the corresponding attractive bands. If 514 we base our comparison between experimental and simulation 515 results on $d$, these systems would correspond to $L_{z} \simeq 5$ and $L_{S} 516$ from 8 to 10 grid spacings in our simulation. As seen in Figures 517 $5 \mathrm{a}$ and $5 \mathrm{~b}$, the structures predicted by our simulation for $L_{z}=6518$ and $L_{S}=8$ and 10 , respectively, agree very well with the 519 experimental results shown in Figure 2 of Park et al. ${ }^{10}$ Hence, 520 the CDS can be compared with physical systems taking the 521 characteristic distance $d$ as a reference.

522

Moreover, this good agreement in a narrow slit indicates that 523 the free-energy functional given in eq 7 is able to reproduce 524 almost quantitatively the experimental results. The kernel of the 525 long-range contribution in eq 7 should contain an explicit 526 dependence on the distance $z$ to the hard walls when $z<d .527$ However, in view of the comparison with experimental results, 528 the effects of such contribution are not relevant, and therefore 529 one can use the isotropic propagator $G(\mathbf{r})$ in all cases, as a good 530 approximation.

We next address the case of a thin slit $L_{z}=8 \simeq 0.7 d$ to 532 analyze the effects of the width of the stripes on the structure of 533 the BCP system. For comparison purposes, let us first consider 534 a nonpatterned homogeneous lower surface for which the 535 boundary conditions are set to be the same as in the upper 536 surface. In general, these neutral boundary conditions favor the 537 formation of semispheres (spherical caps) in contact with the 538 hard walls if no other constraints are applied. Effectively, 539 spherical caps at the surfaces permit the BCP chains close to it 540 to lay unstretched parallel to the wall. Spheres tangent to the 541 hard wall are not entropically favored due to the fact that 542 polymer blocks in the corona shell are forced to squeeze 543 a)

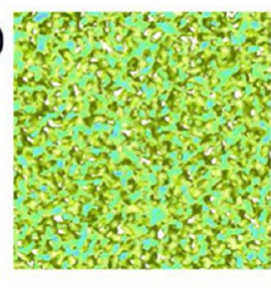

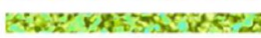

$\mathrm{t}=10$

b)

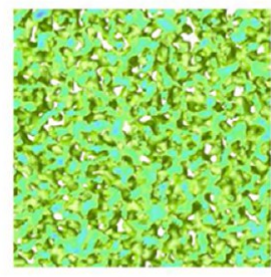

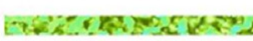

$\mathrm{t}=10$
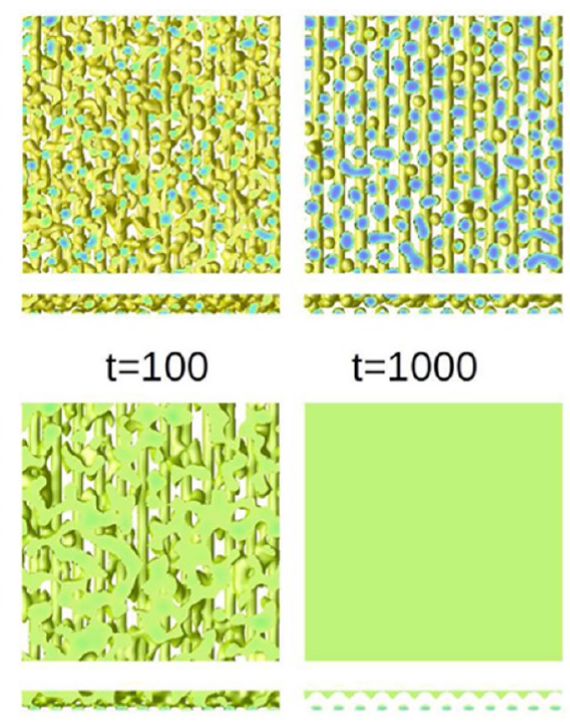

$\mathrm{t}=100$

$$
t=1000
$$

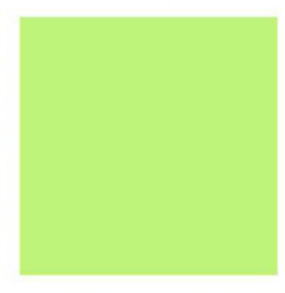

$\mathrm{t}=1000$
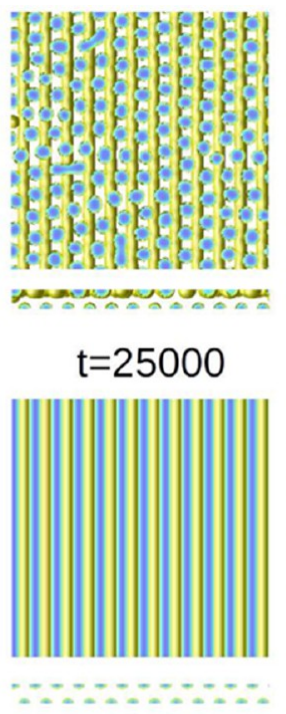

$t=20000$
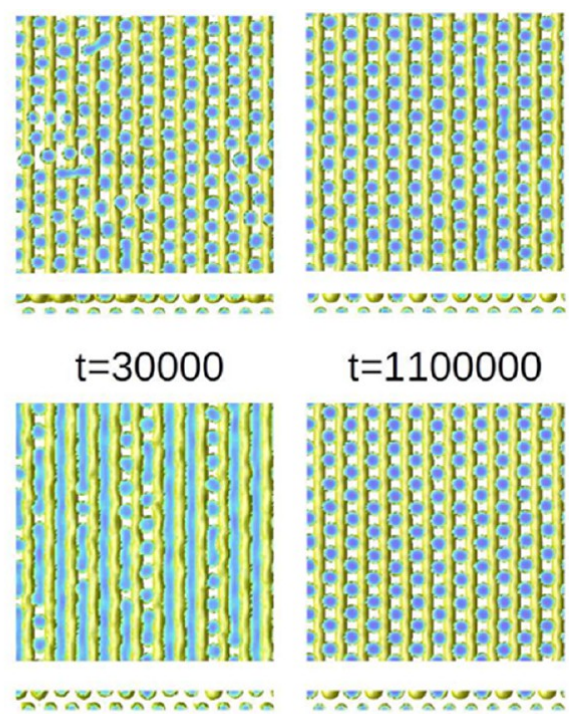

$t=1100000$

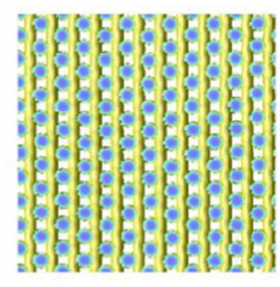

$t=100000$

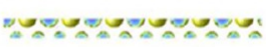

$t=1100000$

Figure 4. Evolution of the configurations of a sphere-forming BCP confined by solid surfaces: (a) quenching and (b) annealing. Box size $L_{x}=L_{y}=$ $120, L_{z}=10$. The square frames show the top view, while the rectangles are side views in the $y$-direction, along which the stripes with $L_{S}=10$ lie. The scale of color ranges from light, $\Psi^{+}=0.25$, to dark, $\Psi^{-}=-1.46$, where $\Psi^{ \pm}$are the minima of $H$. 
544 between the sphere and the wall. This type of confinement of 545 the BCP system therefore produces frustrated situations.

a)

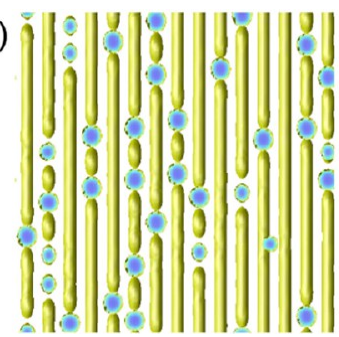

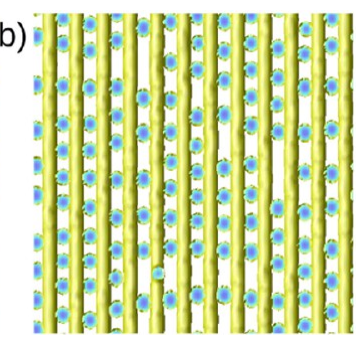

Figure 5. CDS simulations of sphere-forming BCP confined by solid surfaces. Bottom surface chemically activated with stripes attractive to A block and B block alternately and top surface neutral. Box size $L_{x}=$ $L_{y}=120, L_{z}=6$. (a) Top view of the morphology with stripes whose spacing is $L_{S}=8$ and (b) $L_{S}=10$.
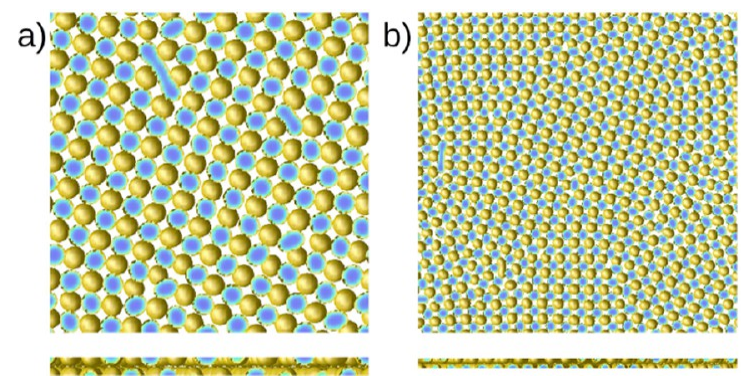

Figure 6. CDS simulations of sphere-forming BCP confined by two homogeneous and neutral solid surfaces. Top and side view of the morphology. Box size: (a) $L_{x}=L_{y}=120, L_{z}=8$ and (b) $L_{x}=L_{y}=240$, $L_{z}=8$.

546 From Figure 6a we observe that two layers of semispheres 547 are formed attached to both the upper and lower surfaces. 548 These semispheres have a tendency to arrange in squares 549 although the figure does not show a clear long-range order. 550 This configuration, however, may not correspond to an 551 equilibrium system. The defects are a signature of a rather 552 degenerated free-energy landscape, since the model we use 553 does not account for thermal fluctuations. The explicit 554 consideration of the noise in this kind of situation could 555 significantly change the phase diagram and the nature of the 556 order-disorder transitions. ${ }^{69,95,96}$ Figure 6 b shows a system 4 557 times the size of the one of Figure $6 \mathrm{a}$, after the same evolution 558 time and from analogous initial conditions. While isolated 559 dislocations are visible in the smaller system, the larger system 560 shows also grain boundaries which become observable due to 561 the less stringent effect of $\mathrm{PBC}$ in the long-range order for the 562 bigger box.

563 It may seem surprising that the dominating structure is 564 square instead of hexagonal, since hard walls tend to favor 565 compact arrangements of hard spheres at their immediate 566 vicinity. However, one should bear in mind that the slit 567 thickness $L_{z}$ is smaller than $d \sqrt{2 / 3} \simeq 9$, which is the distance 568 between layers of spheres in hexagonal packing. Hence, the 569 system chooses to form a square structure that permits the two 570 layers of spheres to interpenetrate with regularity, in a way that 571 a compact hexagonal phase cannot. Notice that the distance 572 between (100) planes of a BCC, whose distance between the 573 central sphere and one at the vertex of the cell is approximately $574 d$, is of the order of $d / \sqrt{3} \simeq 6.3$, which is smaller than the actual $L_{z}=8$. Therefore, the square structure is the result of the 575 need of the system to accommodate two close layers of 576 spherical caps forced by the slit thickness.

577

The presence of the pattern of chemical stripes in the lower 578 surface for the same system $L_{z}=8$ has an additional ordering 579 effect. However, the impact of the pattern on the final 580 configuration of spheres depends upon the commensurability of 581 the periodicity of the stripes $L_{S}$ and the characteristic lengths of 582 the physical system. Let us first consider a pattern with band 583 periodicity $L_{S}=8<L_{0}$, which is approximately equal to the 584 sphere diameter but smaller than the natural distance between 585 spheres, $d \simeq 11$. The structure with lowest energy in this case is 586 given in Figure $7 \mathrm{a}$. On the bottom surface semicylindrical $587 \mathrm{f} 7$

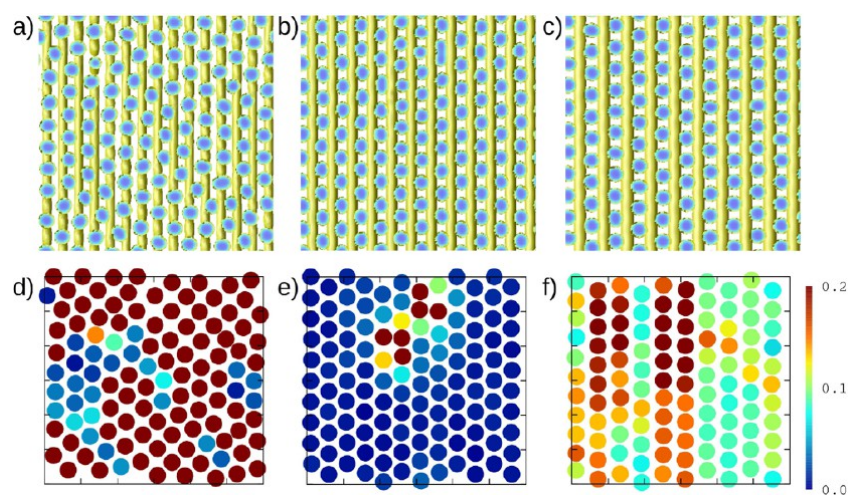

Figure 7. CDS simulations of sphere-forming BCP confined by solid surfaces. Bottom surface chemically activated with stripes attractive to A block and B block alternately and top surface neutral. Box size: $L_{x}=$ $L_{y}=120, L_{z}=8$. (a) Top view of the morphology with stripes whose spacing is $L_{S}=8$, (b) $L_{S}=10$, and (c) $L_{S}=12$. Euclidean distance of the local environments of each spherical domain to the HEX pattern, using a scale of colors. Dark blue indicates a small distance and dark red indicates a large distance to the HEX pattern: (d) $L_{S}=8$, (e) $L_{S}=$ 10 , and (f) $L_{S}=12$.

domains, wetting the A-attracting bands, form. Moreover, a 588 layer of semispheres, attached to the upper surface, also appear. 589 Unlike the nonpatterned case, the bottom layer cannot freely 590 match the structure formed on the upper surface and satisfy the 591 periodicity introduced by the bands. This difference in the 592 periodicity is known as surface reconstruction. Therefore, the 593 addition of the bands causes a change in the native 594 arrangement, as shown in Figure 6, yielding a closer packing 595 of spherical domains than in the nonpatterned case. The 596 structure, however, has many defects, and in some regions 597 square arrangements are still observed. That is mainly because 598 of the mismatch between the horizontal periodicity of the 599 domains in the upper layer, $L_{0} \simeq 9.5$, and the band periodicity, 600 $L_{S}=8$. Simulations of larger system sizes, together with 2-D 601 Fourier transform of the patterns, confirm that there is no long- 602 range order.

From the $L_{S}=8$ case we see that the system has a rather 604 degenerate free-energy landscape. Effectively, in Figure 8a we 605 f8 show the regular structure obtained from the annealing route 606 for the same system. The value of the free-energy for the latter 607 is, however, slightly larger than for the structure in Figure 7a, 608 obtained from quenching. The bottom view of the system, 609 shown in Figure 8b, reveals that the cylinders are broken at the 610 position of the semispheres.

611

For the case $L_{S}=10 \simeq L_{0}$ we match the commensurability 612 between the natural distance for a 2-D HEX structure and the 613 


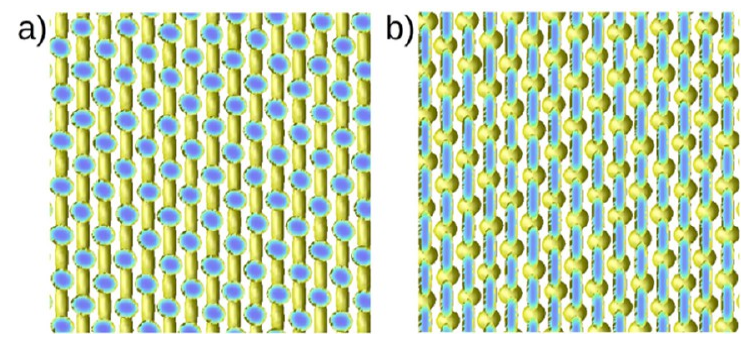

Figure 8. Sphere-forming BCP confined between a homogeneous and neutral surface on the top and a patterned surface on the bottom with chemically active stripes with a separation $L_{S}=8$. State obtained from annealing. (a) Top view and (b) bottom view. Box size: $L_{x}=L_{y}=120$, $L_{z}=8$.

614 periodicity of the stripes, namely $L_{S} \simeq L_{0}$, according to Figure 1 . 615 In Figure $7 \mathrm{~b}$ we observe that in the upper layer an expected 616 regular hexagonal pattern of spherical caps lying in the valley 617 between half-cylinders appears. Finally, the case $L_{S}=12>L_{0}$ 618 yields a regular structure with the spherical caps of the upper 619 surface also lying in the corresponding valleys. However, the 620 structure is forced to comply with the periodicity imposed by 621 the stripes in the $x$-direction. The structure is then slightly 622 stretched yielding a distance between half-spheres belonging to 623 parallel rows approximately $\sqrt{L_{S}{ }^{2}+d^{2} / 4} \simeq 13.2>d$. In turn, 624 the distance between spheres in the same row is slightly smaller 625 than $d$, and no surface reconstruction occur. The resulting 626 structure is then similar to the hexagonal, as seen in Figure $7 \mathrm{c}$. 627 In Figure $7 d-f$ we show for every case the obtained Euclidean 628 distances (cf. Supporting Information) from the local environ629 ments of each spherical domain to the one corresponding to a 630 2-D HEX pattern. For a perfect hexagonal ordering, this 631 distance would be 0 . For the three systems under scrutiny we 632 find that for $L_{S}=8$ only some spherical domains are 633 surrounded by a clear hexagonal pattern; for $L_{S}=10$, Figure $6347 \mathrm{e}$ indicates that the 2-D HEX structure is indeed dominant, 635 except for a few defects; finally, when $L_{S}=12$, the system shows 636 a clear deviation from a hexagonal symmetry, although it is not 637 evident to the eye. We have further checked, for this latter $L_{S}=$ 63812 system, that the Euclidean distance to a (110) plane of a 639 BCC pattern is larger than to the 2-D HEX. Hence, the new 640 structure cannot be considered as a precursor of a bulk BCC. 641 The next case studied is $L_{z}=10 \simeq 0.9$ d. First, the system $L_{S}$ $642=8<L_{0}$ in Figure 9a approximately corresponds to the 643 experimental system with $L_{z}=57 \mathrm{~nm}$ and $L_{S}=50 \mathrm{~nm}$ in Park et 644 al., ${ }^{10}$ where we use $d=11 \approx 65.2 \mathrm{~nm}$ for comparison, as before. 645 Here, the regions with squares, observed for a thickness of $L_{z}=$

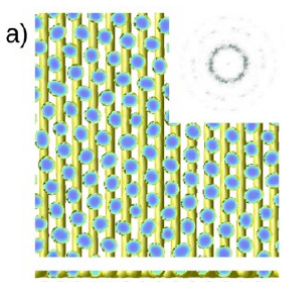

-

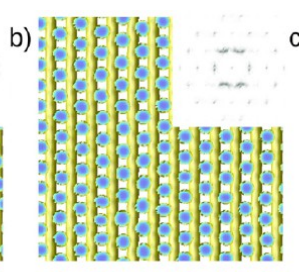

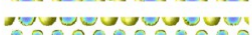

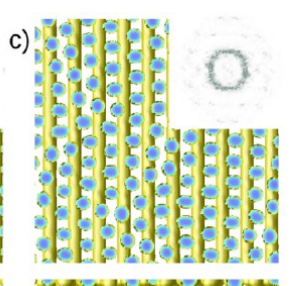

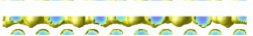

Figure 9. CDS simulations of sphere-forming BCP confined by solid surfaces. Bottom surface chemically activated with stripes attractive to A block and B block alternately and top surface neutral. Top and side view of the morphology when the box size is $L_{x}=L_{y}=120, L_{z}=10$ and the stripes have a spacing of (a) $L_{S}=8$, (b) $L_{S}=10$, and (c) $L_{S}=$ 12. The insets show the corresponding 2-D fast Fourier transform.
8 , have disappeared. The matching between the patterns in the 646 upper and lower layer for this band periodicity is less stringent 647 for a thickness of the order of the natural distance between 648 spherical domains. Then hexagonal structures are observed, 649 although no long-range order exists, in agreement with the 650 experimental results ${ }^{10}$ (see Figure 4 of this last reference). Our 651 CDS data thus confirm that such narrow bands are not capable 652 of guiding the assembled BCP spherical domains toward a 653 regular pattern with long-range order. Again, surface 654 reconstruction occurs as an undesirable result causing the 655 mismatch between the natural spacing of the spheres and the 656 band periodicity.

Second, the system with $L_{S}=10 \simeq L_{0}$ (Figure $9 \mathrm{~b}$ ) produces a 658 well-ordered upper layer of spherical caps. These results are in 659 perfect agreement with the corresponding system $L_{z}=57 \mathrm{~nm} 660$ and $L_{S}=60 \mathrm{~nm}$ described in Figure 4 from Park et al. ${ }^{10}$ As 661 compared with the previous cases, here the system prefers to 662 form a 2-D HEX layer of spherical caps which perfectly matches 663 the periodicity of the stripes of the bottom layer. Figure $10664 \mathrm{f} 10$ shows the system $L_{S}=10$ cropped at $z=4$. One can distinguish 665 knurled cylindrical domains which also reproduce experimental 666 observations. $^{10}$

667
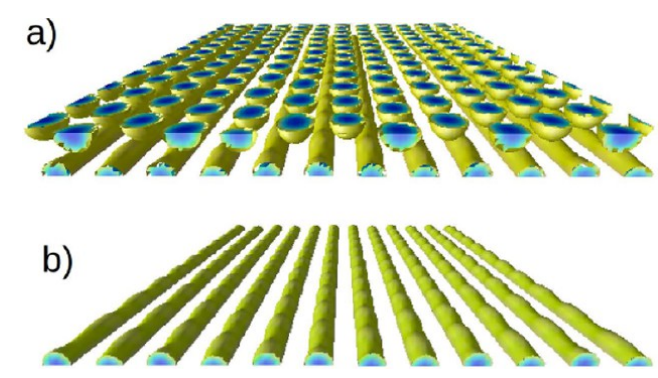

Figure 10. 3-D view of system with $L_{S}=10$ at different heights: (a) at $L_{z}=4$; (b) at $L_{z}=10$.

Beyond the band periodicity experimentally studied we have 668 also analyzed, in the third place, the case $L_{S}=12>L_{0}$ that 669 would physically correspond to a band periodicity of $71 \mathrm{~nm} .670$ Here, this band periodicity matches approximately the length of 671 the edge of a BCC cell, $2 d / \sqrt{3} \simeq 12.7$, where the shortest 672 distance apex center is $d$. However, for this particular slit 673 thickness the bands are not capable of producing a (100) BCC 674 plane on the upper surface, but rather a local HEX-like 675 structure with no long-range order. The comparison of this 676 latter case with $L_{z}=8$ (cf. Figure 9c) suggests that the 677 periodicity in the $x$-direction is not uniquely imposed by the 678 band spacing, unlike suggested elsewhere. ${ }^{10} 679$

It is particularly interesting that a slight increase of the slit 680 thickness from $L_{z}=10$ to 12 for the same $L_{S}=12$ produces a 681 completely different scenario, which is shown in Figure 11. The $682 \mathrm{f} 11$ pattern has long-range order and is rectangular, slightly 683 stretched in the $y$-direction. The new pattern contains an 684 additional layer of complete spheres between the upper and 685 lower layers of half-domains. Then the periodicity of the 686 arrangements of spheres in the $x$-direction perfectly matches 687 the band spacing. The dimensions of the unit cell are estimated 688 as being of 12 lattice spacings in the $x$-direction and of 13.3 in 689 the $y$-direction, parallel to the stripes. The unit cell is then a 690 body-centered orthorhombic (BCO).

Slit $L_{z}=14$. We also investigated the morphology of 692 systems contained in a slit with thickness $L_{z}=14 \simeq 1.3 d$. The 693 


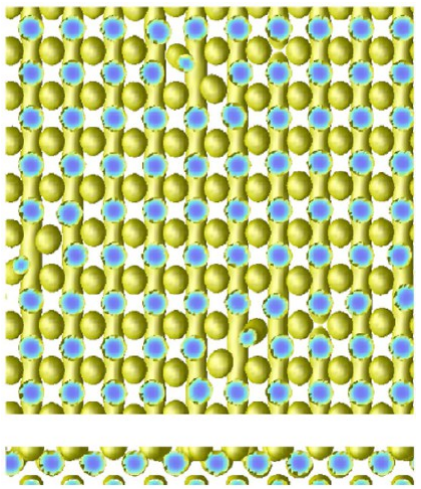

Figure 11. CDS simulations of sphere-forming BCP confined by solid surfaces. Bottom surface chemically activated with stripes attractive to A block and B block alternately and top surface neutral. Top and side view of the morphology when the box size is $L_{x}=L_{y}=120, L_{z}=12$ and the stripes have a spacing of $L_{S}=12$.

694 spherical caps forming 2-D HEX pattern of the previous $L_{z}=$ 69510 case are transformed in a layer of complete spheres almost 696 tangent to the hard wall, which is entropically unfavorable. The 697 system is therefore frustrated if 2-D HEX layers are to be 698 formed in a slit where $L_{z}$ is slightly larger than $d$.

699 The first case $L_{S}=8<L_{0}$ produces complete tangent spheres 700 locally ordered in hexagonal arrangements in addition of the 701 half-cylinders formed on the lower hard wall, according to 702 Figure 12a. Some of the domains in the upper layer, however,

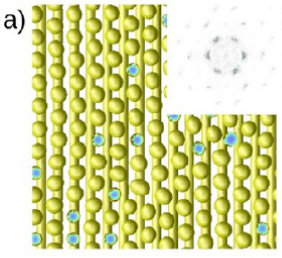

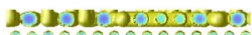
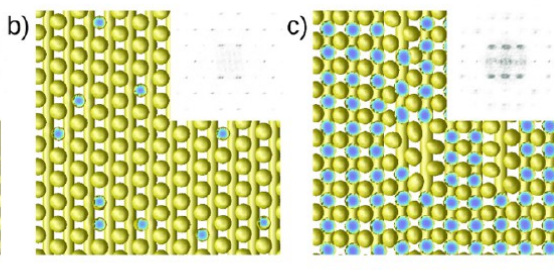

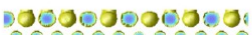

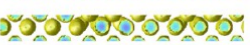

Figure 12. CDS simulations of sphere-forming BCP confined by solid surfaces. Bottom surface chemically activated with stripes attractive to A block and B block alternately and top surface neutral. Top and side view of the morphology when the box size is $L_{x}=L_{y}=120, L_{z}=14$ and the stripes have a spacing of (a) $L_{S}=8$, (b) $L_{S}=10$, and (c) $L_{S}=$ 12. The insets show the corresponding 2-D fast Fourier transform.

703 are deformed into structures that vaguely resemble eggplants, 704 due to the aforementioned frustration. The overall behavior is 705 very similar to the previously studied case $L_{z}=10$ for the same 706 band periodicity. We observe here again surface reconstruction 707 with lost of long-range order in larger systems (not shown). 708 Hence, bands cannot guide the assembly of the upper layer of 709 spheres.

710 As expected, for the case $L_{S}=10$ there is a clear effect of the 711 commensurability, since $L_{S} \simeq L_{0}$, in view of Figure $12 \mathrm{~b}$. The 712 arrangement obtained corresponds to upper layer of complete 713 spheres laying tangent to the hard wall, with a long-range 2-D 714 HEX symmetry. Some defects in the form of eggplant domains 715 are also present, and the distribution of domains in the upper 716 layer is similar to the previous cases studied for this band 717 periodicity. Our simulation results are in agreement with the 718 patterns encountered in the system $L_{S}=60 \mathrm{~nm}$ in a slit $L_{z}=70$ $719 \mathrm{~nm},{ }^{10}$ although we find a slightly better long-range ordered 720 structures.
Finally, the most interesting situation occurs for the band 721 periodicity to $L_{S}=12>L_{0}$. Figure $12 \mathrm{c}$ shows an extra layer of 722 domains that are formed in contact with the upper wall, as 723 already encountered for the case $L_{z}=12$ with the same band 724 spacing. The order is long-range and corresponds to BCO with 725 the (100) plane parallel to the hard surfaces. This is a clear 726 example of the interplay of both the band periodicity and the 727 slit thickness to favor a given 3-D cell. In this particular case, a 728 $\mathrm{BCO}$ is favored due to the fact that the slit thickness is close to 729 $2 d / \sqrt{3} \simeq 12.7$ and that the band periodicity is precisely close to this same length.

730

Thicker Slits. In the previously analyzed cases we have 731 verified that the sphere-forming BCP are very sensitive to the 732 band periodicity, which should be commensurate with the 733 characteristics distance $d$ of the BCP in order to produce neat 734 ordered arrangements. We have seen that the vertical 735 confinement also plays an important role in determining the 736 final structure. In this section we attempt to rationalize these 737 two ideas for systems where many layers of spheres can form. 738

If the system forms $n$ layers of complete spheres stacked in a 739 3-D HEX packing, then the appropriate slit thickness should be 740 approximately given by

$$
L_{z}\left(\operatorname{HEX}_{1}\right) \equiv(n+1) d \sqrt{\frac{2}{3}}
$$

We have considered that the distance between neighboring 743 spheres is approximately $d$ and that the distance between layers 744 parallel to the walls is $d \sqrt{2 / 3}$, corresponding to a 3-D HEX 745 packing of hard spheres of a diameter $d$. An additional layer of 746 spherical caps forms in contact with the top surface. The 747 distance between the half-cylinders at the lower surface and the 748 first layer of spheres has been assimilated to the distance 749 between neighboring planes of spheres.

750

In the same way, if the system is prone to form a BCC unit 751 cell with its base parallel to the hard walls, the appropriate slit 752 thickness should be approximately given by

753

$$
L_{z}(\mathrm{BCC}) \equiv(n+1) \frac{d}{\sqrt{3}}
$$

Here, the distance between layers is $d / \sqrt{3}$, as it corresponds to 755 (100) planes of a BCC. Again, a layer of spherical caps is 756 considered to form on the upper wall while the shortest 757 distance between neighboring spheres is assumed to be 758 approximately $d$.

759

Slit thicknesses laying off these periodicities impose some 760 degree of frustration in the system, which may produce slightly 761 stretched or compressed unit cells, or the formation of 762 entropically unfavorable tangent spheres near the upper wall. 763 In particular, a slit thickness given by

$$
L_{z}\left(\operatorname{HEX}_{2}\right) \equiv\left(n+\frac{1}{2}\right) d \sqrt{\frac{2}{3}}
$$

with 3-D HEX packing would produce such a frustrated system 766 with tangent spheres. Equations 24-26 cannot be taken as 767 exact by different reasons, the most important being that the 768 system can modify its characteristic length under constraint to a 769 given extent. These expressions only give a rough estimate of 770 what one can expect.

In the first place, let us study the structures obtained for the 772 three band periodicities $L_{S}=8,10$, and 12 for systems with slit 773 thicknesses $L_{z}=20,24$, and 36 (corresponding to $1.8 d, 2.2 d, 774$ 
a)

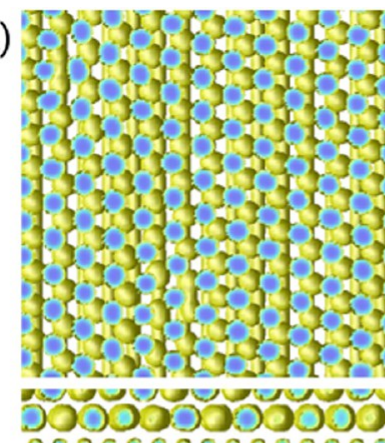

\section{d)}

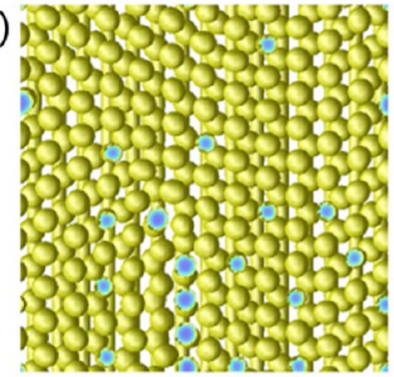

1004055000001 000030000000

g)

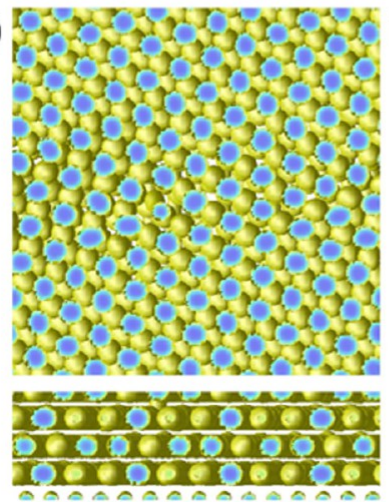

b)

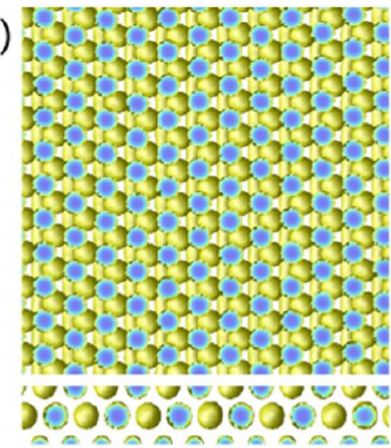

e)

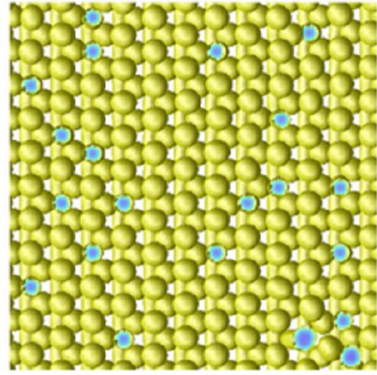

100000000005

200000000100

h)

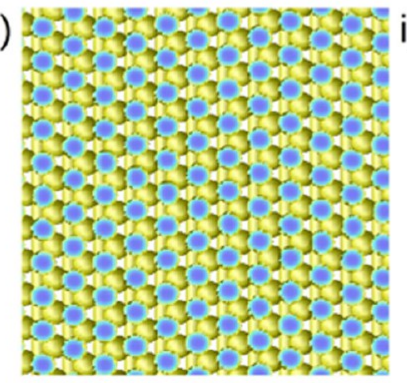

00000000 00000000000 2000000000
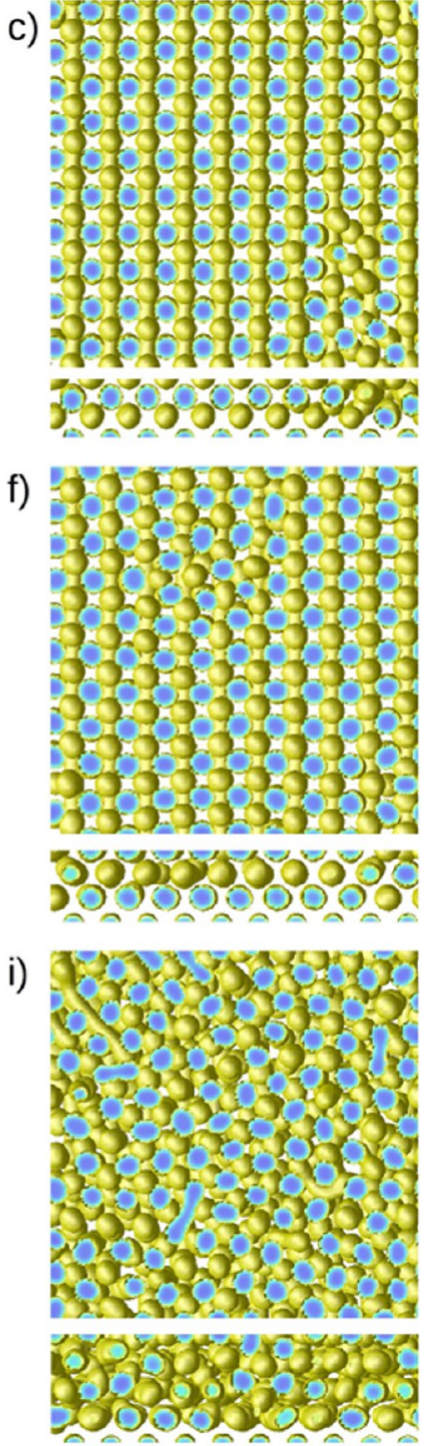

Figure 13. CDS simulations of sphere-forming BCP confined by solid surfaces. Bottom surface chemically activated with stripes attractive to A block and B block alternately and top surface neutral. Top and side view of the morphology when the box size is $L_{x}=L_{y}=120, L_{z}=20$ and the stripes have a spacing of (a) $L_{S}=8$, (b) $L_{S}=10$, and (c) $L_{S}=12$. Top view for a box size $L_{x}=L_{y}=120, L_{z}=24$ and lattice spacing of the stripes (d) $L_{S}=8$, (e) $L_{S}=10$, and (f) $L_{S}=12$. Top view for a box size $L_{x}=L_{y}=120, L_{z}=36$ and lattice spacing of the stripes $(\mathrm{g}) L_{S}=8$, (h) $L_{S}=10$, and (i) $L_{S}=12$.

775 and $3.3 d$, respectively). The particular thicknesses chosen for 776 the first and the third cases correspond to values that 777 approximately comply with eq 24 , with $n=1$ and $n=3$. The 778 second thickness roughly corresponds to eq 26 with $n=2$. In 779 Figure 13 we show the structures obtained for the mentioned 780 periodicities and thicknesses.

781 First, let us center our attention to the case of a bandwidth $L_{S}$ $782=8<L_{0}$. Here, the structure is dominated by the geometrical 783 confinement. Figures 13a,g show a 2-D hexagonal pattern in the 784 upper layer, with spherical caps. Figure $13 \mathrm{~d}$ shows the same 785 structure but for tangent spheres. The mismatch between the 786 bandwidth and the 2-D HEX pattern is seen in the lateral views 787 of the systems in the same set of figures. Although the system 788 shows local order, many defects in the structure are visible for 789 the three thicknesses. Surface reconstruction is also visible.

790 Second, Figures $13 \mathrm{~b}, \mathrm{e}, \mathrm{h}$ correspond to a band periodicity $L_{S}$ $791=10 \simeq L_{0}$, compatible with the formation of a 2-D hexagonal 792 pattern in the first layer of spheres (bottom up). For the three 793 thicknesses, we observe long-range order in all the layers. From
$L_{z}=36$ we further see that the three layers of complete spheres 794 together with the upper layer of spherical caps are packed in a 795 clear 3-D HEX, with a distance between layers of spheres 796 $\delta \simeq 9 \simeq d \sqrt{2 / 3}$. Effectively, from the top view of Figure $13 \mathrm{~h}$ we see that the top layer of spherical caps and the second layer 797 bottom up are on top of each other, as it corresponds to this 3- 798 D HEX structure (see also the lateral view). The calculation of 799 the Euclidean distance at each layer of spheres also reveals that 800 the local structures are indeed hexagons (Euclidean distances to 801 the reference pattern are of the order of $10^{-2}$ ). The case $L_{z}=24802$ corresponds to tangent spheres. The system keeps the overall 803 3-D HEX configuration despite the fact that tangent spheres are 804 entropically less favored. It is worth remarking that the distance 805 between planes for this latter case is $\delta \simeq 9.6$ instead of 9,806 slightly stretched with respect to a 3-D HEX packing. $\quad 807$

Third, Figures $13 \mathrm{c}, \mathrm{f}, \mathrm{i}$ correspond to a bandwidth $L_{S}=12>808$ $L_{0}$. With this constraint on the first layer, the system builds a 809 $\mathrm{BCO}$, with a distance between planes $\delta \simeq 6.1$ for the thickness 810 $L_{z}=20$, which is very close to the separation between (100) 811 

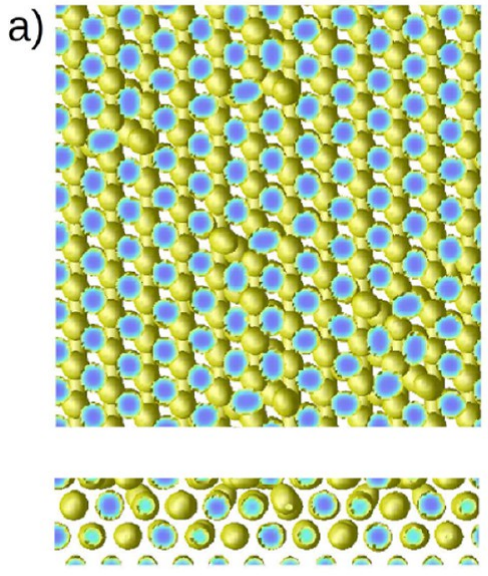
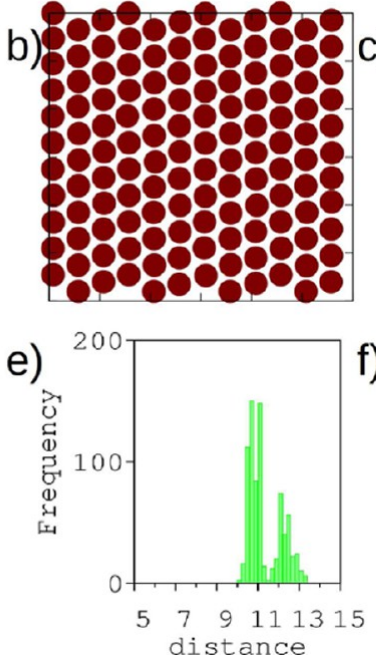
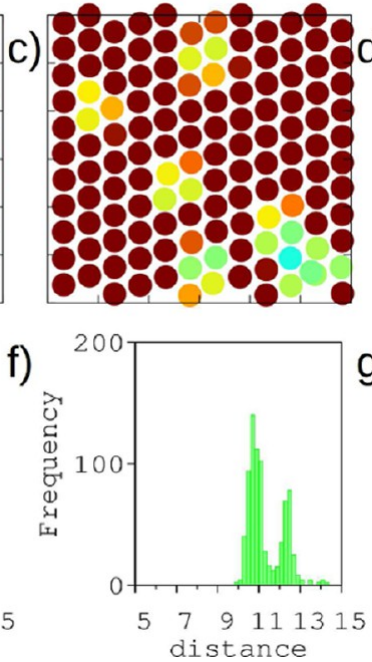

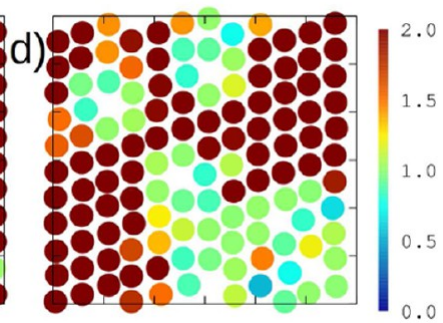

g)

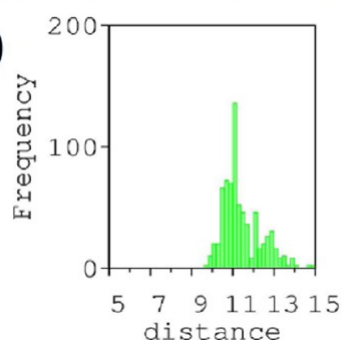

Figure 14. CDS simulations of sphere-forming BCP confined by solid surfaces. Bottom surface chemically activated with stripes attractive to A block and B block alternately and top surface neutral. Box size $L_{x}=L_{y}=120, L_{z}=26$ and stripes with a spacing of $L_{S}=10$. (a) Top and side view of the morphology. Ratio of the Euclidean distance to HEX symmetry and distance to BCC symmetry $\left(d_{\mathrm{HEX}} / d_{\mathrm{BCC}}\right)$; (b) lower layer; (c) middle layer; (d) upper layer. Histogram of the distances of each sphere with its six next-nearest neighbors: (e) lower layer, (f) middle layer, and ( $\mathrm{g}$ ) upper layer.

812 planes of the BCC lattice, namely, $\delta=d / \sqrt{3} \simeq 6.35$. For the 813 thickness $L_{z}=24$, the separation is slightly larger $\delta \simeq 7.7$, as if 814 the system were stretched in the $z$-direction. These two cases 815 present overall long-range order although some defects are 816 visible. However, in Figure 13i we see that the system shows no 817 local order due to the mismatch between the side length of the 818 BCC unit cell and the slit thickness.

819 Before closing the analysis of the results, several comments 820 are in order. It is well-known that the sphere-forming BCP 821 organize in bulk in BCC structure. ${ }^{11}$ The transition between 822 the 2-D hexagonal morphology near a nonpatterned surface 823 and the bulk BCC cell has been studied by Stein et al. ${ }^{45,97}$ 824 These authors find a first-order transition at $n \approx 4$ between a 3825 D HEX and a face-centered orthorhombic unit cell, which 826 smoothly tends to the bulk BCC as the layer lays farther from 827 the hard surface. Our simulations for $L_{S}=10$, which favors the 828 3-D HEX pattern, thus agree with this experimental finding, 829 particularly when the slit thickness corresponds to eq 24 , and 830 therefore, the possible distorting effect of the upper hard wall is 831 minimized. We have found, however, deviations from the 832 expected behavior of 3-D HEX packing in $L_{z}=26 \simeq 2.4 d$, only 833 slightly thicker than the case $L_{z}=24 \simeq 2.2 d$, with the same 834 band periodicity $L_{S}=10$. Here the slit thickness is close to but 835 smaller than the one given in eq 24 with $n=2$ and is therefore 836 frustrated. Notice that with respect to the case $L_{z}=24$, where 837 the upper layer is tangent to the upper wall, the system $L_{z}=26$ 838 forms an additional layer of spherical caps, but not completely 839 developed, as shown in Figure 14a. In Figures 14b-d, we show 840 the ratio between the Euclidean distances of the local structure 841 to both the 2-D HEX and the (110) plane of a BCC. Large 842 values of this ratio indicate that the local environment is closer 843 to the (110) plane of BCC than to the 2-D HEX. Despite the 844 fact that the pattern of the lower surface favors the formation of 845 hexagons, the first layer (bottom up) displays a domain 846 arrangement significantly closer to the (110) BCC than to the 847 HEX. The second layer, however, shows some more disperse 848 spheres surrounded by a HEX environment, while the upper 849 layer of spherical caps is more hexagonal. Obviously, the upper 850 hard wall also imposes a strong confinement constraint that 851 induces a major packing in this layer, favoring the 2-D HEX.
However, it is remarkable that the system shows a body- 852 centered structure, close to the bulk BCC, near the lower 853 surface, without passing through intermediate forms of the type 854 of face-centered orthorhombic, as it has been previously 855 reported. $^{45,97}$ For completeness, in Figures $14 \mathrm{e}-\mathrm{g}$ we show 856 the histograms of the distances between every sphere in a given 857 layer to its six closest neighbors. Two peaks are clearly 858 distinguished corresponding to the two characteristic distances 859 of the (110) BCC plane, in the first and second layer. The ratio 860 $a_{2} / a_{1}$ between these two main distances is the order parameter 861 used to describe the transition from HEX to BCC in the 862 aforementioned references. From the histograms we approx- 863 imately obtain $a_{1} \simeq 10.8$ and $a_{2} \simeq 12.4$, yielding $a_{2} / a_{1} \simeq 1.15$, 864 which confirms that the layer indeed corresponds to a (110) 865 plane of a BCC. One can then argue that the formation of 866 partial spherical caps on the upper surface compresses the 867 structure in the $z$-direction. Effectively, we measure a distance 868 between planes $\delta \simeq 8$ instead of 9 , as would correspond for 869 both the 3-D HEX and the (110) BCC as well. Therefore, the 870 structure is not cubic but rather a body-centered rhombohe- 871 dron (BCR), instead of the previously observed BCO, due to 872 this compression. It is quite surprising, however, that the 873 surface density of spheres is insensitive to such compression. 874 The case $L_{z}=24$ instead displays a clear 3-D HEX packing of 875 the layers. This can be observed from the ratio of the Euclidean 876 distances as well as from the histograms in Figure 15. These $877 \mathrm{f} 15$ behaviors are rather counterintuitive, since the 3-D HEX 878 permits a larger packing density than a BCC for hard spheres 879 and therefore should apparently be preferred in systems under 880 pressure.

A second frustrated system with $L_{S}=10$ is the case $L_{z}=34 \simeq 882$ $3.1 d$, which corresponds to a slit thickness complying with the 883 formation of three layers of complete spheres, but not thick 884 enough to form spherical caps. Hence, we have an upper layer 885 of tangent spheres with some eggplant defects. The interesting 886 feature of this system is that within the planes the domains 887 adopt an 2-D HEX packing, but when analyzing the 3-D 888 packing, one observes that is not 3-D HEX, but closer to a 889 (111) plane of face-centered cubic phase (FCC), since the first 890 and the third layer are not superposed in the $x y$-plane. The 891 

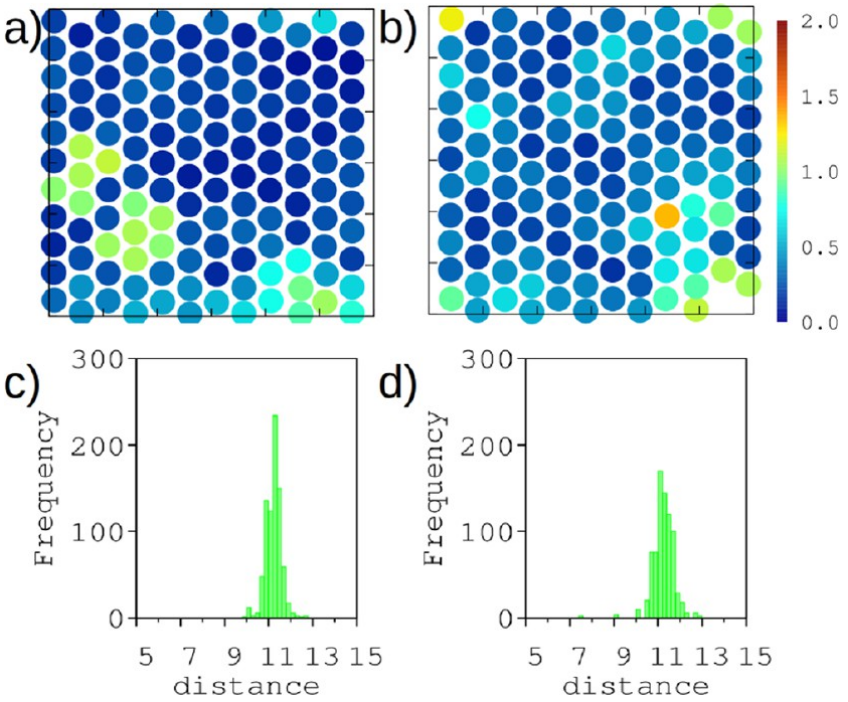

Figure 15. CDS simulations of sphere-forming BCP confined by solid surfaces. Bottom surface chemically activated with stripes attractive to A block and B block alternately and top surface neutral. Box size $L_{x}=$ $L_{y}=120, L_{z}=24$ and stripes with a spacing of $L_{S}=10$. Ratio of the Euclidean distance to HEX symmetry and distance to BCC symmetry $\left(d_{\mathrm{HEX}} / d_{\mathrm{BCC}}\right)$ : (a) lower layer; (b) upper layer. Histogram of the distances of each sphere with its six next-nearest neighbors: (d) lower layer; (e) upper layer.

892 packing density of both 3-D structures is, however, the same. 893 Thus, the reason for the existence of this new structure could 894 be that the equilibrium for such thickness is degenerated with 895 two possible structures of maximum packing, namely, 3-D HEX 896 and (111) FCC.

897 We have also analyzed systems of $L_{z}=54 \simeq 4.9 d$ and $56 \simeq$ $8985.1 d$ with the band periodicity $L_{S}=10 \simeq L_{0}$, capable of 899 allocating five layers of complete spheres, tangent to the upper 900 wall or with spherical caps in it, respectively. The 3-D HEX 901 order is maintained in the $L_{z}=56$, with a plane separation 902 approximately $\delta=9.3$. As before, the system with tangent 903 spheres, $L_{z}=54$, produces (111) planes of a face-centered 904 body, with $\delta \simeq 9.8$, and many eggplant defects at the upper 905 layer. However, for $L_{S}=12$ we obtain disordered systems for $n$ $906 \geq 4$ layers of complete spheres, even with the appropriate $L_{z}$ as 907 given in eq 25 , from both quenching and annealing routes.

\section{CONCLUSIONS}

908 In this article we have shown that the CDS is a powerful tool to 909 predict the self-assembly of block copolymers into complex 910 structures. Simulations of sphere-forming BCP on patterned 911 surfaces confined in a slit have been compared with 912 experimental data from Park et al., ${ }^{10}$ and an almost quantitative 913 agreement is obtained. The comparison is done in terms of 914 only one single characteristic length, namely, $d$, which is known 915 to be physically proportional to a power of the size of the BCP $916 d \sim N^{2 / 3}$, in the strong segregation limit. The size of the 917 domains of a given block seems to play a secondary role, 918 provided that the domain geometry keeps being spherical.

919 Because of this inner simplicity of the problem, the structures 920 that can be formed are rather limited by the bulk BCP 921 equilibrium configuration and the hexagonal packing produced 922 by the presence of a hard wall. The main result of this article 923 lies in the fact that by tuning the band spacing of the chemically 924 patterned surfaces together with the slit thicknesses, one of these two arrangements can be selected and produce long- 925 range order structures, at least of the order of the size of the 926 simulated systems. Along with the minimal distance between 927 domains $d$, the system is sensitive to the band periodicity if the 928 aim is at promoting a particular 3-D structure. In this way, if the 929 band periodicity is approximately $L_{0}=d \sqrt{3} / 2$, the first layer 930 of complete spheres (bottom up) is naturally hexagonal. It is 931 additionally required, however, that the slit thickness is 932 appropriate for a 3-D hexagonal cell to develop without 933 conflict with upper surface constraint, as described in eq 24. On 934 the contrary, if the band periodicity is approximately $2 d / \sqrt{3} 935$ and the slit thickness is approximately given by eq 25 , the 936 system naturally develops a BCO structure already from the 937 first layer. The latter result is a pure prediction of the CDS 938 since no experiments have been done under these conditions 939 yet, to the best of our knowledge. This fact is significantly 940 different from what occurs in the presence of a nonpatterned 941 lower surface, where the confining effect of the hard wall 942 imposes a preference for a hexagonal structure.

943

We have also analyzed frustrated situations to explore the 944 possibility of existence of exotic structures generated by the 945 constraints, for the same physical system. On one hand, we 946 have encountered hexagonally packed ordered systems with 947 spheres tangent to the upper wall, formed on surface patterns 948 with band periodicity $L_{S}=L_{0}$, by tuning the slit thickness to 949 approximately that of eq 26. This type of situation has been also 950 observed in the experiments. For this type of frustration, the 951 layer separation $\delta>d \sqrt{2 / 3}$ as it would correspond to the 952 perfect 3-D HEX. This effect can be caused by the presence of 953 eggplant defects, which are deformed domains cut by the upper 954 wall, to favor the conformation of the BCP chains near the wall, 955 pulling the whole system up. On the other hand, we also have 956 studied systems whose thickness is in between the values given 957 by eqs 24 and 26, both with $n=2$ layers of complete spheres. 958 We have analyzed only the case $L_{S}=L_{0}$. We have observed that 959 the first and second layers have a clear structure of a (110) 960 plane of a BCC instead of an expected HEX, probably due to a 961 subtle effect of the bands on the possible displacements of the 962 spheres under changing external conditions, which deserves 963 further investigation. This case shows a distance between the 964 layers slightly compressed $\delta<d \sqrt{2 / 3}$, which can be attributed 965 to the presence of incomplete spheres, cut by the wall between 966 its pole and equator, that push the whole system down. Hence, 967 the fine-tuning of the slit thickness is also of crucial importance 968 to obtain the desired ordered 3-D structures.

969

The analysis carried out in this article suggests that the use of 970 chemical masks on surfaces, together with the control of the 971 thickness can be a useful tool to create the desired structure 972 from the self-assembly of BCP. We believe that this is a step 973 forward toward the applications of this systems in well- 974 controlled fabrication of nanoscopic devices.

\section{ASSOCIATED CONTENT}

The Supporting Information is available free of charge on the 978 ACS Publications website at DOI: 10.1021/acs.macro- 979 mol.5b02314.

Qualitative analysis of the system and Euclidean distance 981 between structures (ZIP) 


\section{$983 \square$ AUTHOR INFORMATION}

\section{Corresponding Authors}

985 *E-mail: josep.bonet@urv.cat (J.B.A.).

986 *E-mail: mpinna@lincoln.ac.uk (M.P.).

987 Notes

988 The authors declare no competing financial interest.

\section{ACKNOWLEDGMENTS}

990 M.S. thanks the Spanish Ministrerio of Educación, Cultura y 991 Deporte (Grant AP2010-2150). Moreover, M.S. thanks A.Z. 992 and M.P. for the hospitality during her stay at the Computa993 tional Physics group, which includes the access to the CDS 994 code and resources during part of the research stay, as well as 995 the scientific assistance of the members of the group, where this 996 research was initiated. M.S. and J.B.A. are grateful for that.

\section{$997-$ REFERENCES}

998 (1) Fasolka, M. J.; Mayes, A. M. Annu. Rev. Mater. Res. 2001, 31, 323.

999 (2) Hamley, I. W. Nanotechnology 2003, 14, R39-R54.

1000 (3) Park, C.; Yoon, J.; Thomas, E. L. Polymer 2003, 44, 6725.

1001 (4) Muller, M.; Sun, D. Phys. Rev. Lett. 2013, 111, 267801.

1002 (5) Park, M.; Harrison, C.; Chaikin, P. M.; Register, R. A.; Adamson, 1003 D. Science 1997, 276, 1401.

1004 (6) Thurn-Albrecht, T.; Schotter, J.; Kastle, G.; Emley, N.; 1005 Stubauchui, T.; Krusin-Elbaum, L.; Black, C.; Tuominen, M.; 1006 Russell, T. Science 2000, 290, 2126.

1007 (7) Segalman, R. A. Mater. Sci. Eng., R 2005, 48, 191.

1008 (8) Hamley, I. W. Prog. Polym. Sci. 2009, 34, 1161-1210.

1009 (9) Kim, J. K.; Yang, S. Y.; Lee, Y.; Kim, Y. Prog. Polym. Sci. 2010, 35, $10101325-1349$.

1011 (10) Park, S.-M.; Craig, G. S. W.; La, Y.-H.; Nealey, P. F. 1012 Macromolecules 2008, 41, 9124-9129.

1013 (11) Bates, F. S.; Fredrickson, G. H. Annu. Rev. Phys. Chem. 1990, 41, 1014525.

1015 (12) Matsen, M. W.; Bates, F. S. Macromolecules 1996, 29, 109110161098

1017 (13) Hamley, I. W. The Physics of Block Copolymers; Oxford 1018 University Press: Oxford, 1998.

1019 (14) Fredrickson, G. H. Macromolecules 1987, 20, 2535-2542.

1020 (15) Wang, Q. In Nanostructured Soft Matter: Experiment, Theory, 1021 Simulation, and Perspectives; Zvelindovsky, A. V., Ed.; Springer: 1022 Dordrecht, 2007; pp 495-528.

1023 (16) Shi, A.-C.; Li, B. In Polymer Science: A Comprehensive Reference; 1024 Matyjaszewski, K., Muller, M., Eds.; Elsevier: Amsterdam, 2012; pp $102571-81$.

1026 (17) Shin, K.; Xiang, H.; Moon, S. I.; Kim, T.; McCarthy, T. J.; 1027 Russell, T. P. Science 2004, 306, 76.

1028 (18) Wu, Y.; Chen, G.; Katsov, K.; Sides, S. W.; Wang, J.; Tang, J.; 1029 Fredrickson, G. H.; Moskovits, M.; Stucky, G. D. Nat. Mater. 2004, 3, 1030816.

1031 (19) Dobriyal, P.; Xiang, H.; Kazuyuki, M.; Chen, J.-T.; Jinnai, H.; 1032 Russell, T. P. Macromolecules 2009, 42, 9082-9088.

1033 (20) Wang, Y.; Qin, Y.; Berger, A.; Yau, E.; He, C.; Zhang, L.; Gosele, 1034 U.; Knez, M.; Steinhart, M. Adv. Mater. 2009, 21, 2763-2766.

1035 (21) Arsenault, A. C.; Rider, D. A.; Tetreault, N.; Chen, J. I.-L.; 1036 Coombs, N.; Ozin, G. A.; Manners, I. J. Am. Chem. Soc. 2005, 127, 1037 9954-9955.

1038 (22) Kellogg, G. J.; Walton, D. G.; Mayes, A. M.; Lambooy, P.; 1039 Russell, T. P.; Gallagher, P. D.; Satija, S. K. Phys. Rev. Lett. 1996, 76, 10402503.

1041 (23) Koneripalli, N.; Levicky, R.; Bates, F. S.; Ankner, J.; Kaiser, H.; 1042 Satija, S. K. Langmuir 1996, 12, 6681-6690.

1043 (24) Huang, E.; Russell, T. P.; Harrison, C.; Chaikin, P. M.; Register, 1044 R. A.; Hawker, C. J.; Mays, J. Macromolecules 1998, 31, 7641.

1045 (25) Knoll, A.; Horvat, A.; Lyakhova, K. S.; Krausch, G.; Sevink, G. J. 1046 A.; Zvelindovsky, A. V.; Magerle, R. Phys. Rev. Lett. 2002, 89, 035501.
(26) Yokoyama, H.; Mates, T. E.; Kramer, E. J. Macromolecules 2000, 1047 33, 1888-1898. 1048

(27) Segalman, R. A.; Hexemer, A.; Kramer, E. J. Phys. Rev. Lett. 1049 2003, 91, 196101. 1050

(28) Angelescu, D. E.; Harrison, C. K.; Trawick, M. L.; Register, R. 1051 A.; Chaikin, P. M. Phys. Rev. Lett. 2005, 95, 025702.

(29) Kramer, E. J. Nature 2005, 437, 824-825. 1053

(30) Li, W.; Muller, M. Annu. Rev. Chem. Biomol. Eng. 2015, 111, 1054 267801.

1055

(31) Kim, H.-C.; Hinsberg, W. D. J. Vac. Sci. Technol., A 2008, 26, 1056 $1369-1382$.

1057

(32) Rockford, L.; Liu, Y.; Mansky, P.; Russell, T. P.; Yoon, M.; 1058 Mochrie, S. Phys. Rev. Lett. 1999, 82, 2602.

(33) Yang, X. M.; Peters, R. D.; Nealey, P. F.; Solak, H. H.; Cerrina, 1060 F. Macromolecules 2000, 33, 9575-9582. 1061

(34) Kim, S. O.; Solak, H. H.; Stoykovich, M. P.; Ferrier, N. J.; Pablo, 1062

J. J.; Nealey, P. F. Nature 2003, 424, 411.

(35) Edwards, E. W.; Muller, M.; Stoykovich, M. P.; Solak, H. H.; de 1064 Pablo, J. J.; Nealey, P. F. Macromolecules 2007, 40, 90-96. 1065

(36) Kim, H.; Daniels, E. S.; Li, S.; Mokkapati, V. K.; Kardos, K. J. 1066 Polym. Sci., Part A: Polym. Chem. 2007, 45, 1038-1054. 1067

(37) Ruiz, R.; Kang, H.; Detcheverry, F. A.; Dobisz, E.; Kercher, D. 1068 S.; Albrecht, T. R.; de Pablo, J. J.; Nealey, P. F. Science 2008, 321, 936. 1069

(38) Tada, Y.; Akasaka, S.; Yoshida, H.; Hasegawa, H.; Dobisz, E.; 1070

Kercher, D.; Takenaka, M. Macromolecules 2008, 41, 9267-9276. 1071

(39) Ji, S.; Nagpal, U.; Liao, W.; Liu, C.-C.; de Pablo, J. J.; Nealey, P. 1072

F. Adv. Mater. 2011, 23, 3692-3697. 1073

(40) Park, S.-M.; Craig, G. S. W.; La, Y.-H.; Solak, H. H.; Nealey, P. 1074

F. Macromolecules 2007, 40, 5084.

(41) Segalman, R.; Yokoyama, H.; Kramer, E. J. Adv. Mater. 2001, 13, 1076

1152-1155. 1077

(42) Chuang, V. P.; Cheng, J. Y.; Savas, T. A.; Ross, C. A. Nano Lett. 1078 2006, 6, 2332-2337.

(43) Bita, I.; Yang, J. K. W.; Jung, Y. S.; Ross, C. A.; Thomas, E. L.; 1080 Berggren, K. K. Science 2008, 321, 939.

(44) Xiao, S.; Yang, X.; Park, S.; Weller, D.; Russell, T. P. Adv. Mater. 1082 2009, 21, 2516-2519. 1083

(45) Stein, G. E.; Kramer, E. J.; Li, X.; Wang, J. Macromolecules 2007, 1084 40, 2453-2460. 1085

(46) Wu, M. W.; Register, R. A.; Chaikin, P. M. Phys. Rev. E 2006, 74, 1086 040801(R).

(47) Xu, J.; Park, S.; Wang, S.; Russell, T. P.; Ocko, B. M.; Checco, A. 1088 Adv. Mater. 2010, 22, 2268.

(48) Ye, X.; Edwards, B. J.; Khomami, B. Macromol. Rapid Commun. 1090 2012, 33, 392-395. 1091

(49) Ramírez-Hernández, A.; Liu, G.; Nealey, P. F.; de Pablo, J. J. 1092 Macromolecules 2012, 45, 2588-2596. 1093

(50) Liu, G.; Detcheverry, F.; Ramírez-Hernández, A.; Yoshida, H.; 1094 Tada, Y.; de Pablo, J. J.; Nealey, P. F. Macromolecules 2012, 45, 3986- 1095 3992.

(51) Chen, P.; Liang, H.; Xia, R.; Qian, J.; Feng, X. Macromolecules 1097 2013, 46, 922-926.

(52) Liu, C.-C.; Ramírez-Hernández, A.; Han, E.; Craig, G. S. W.; 1099 Tada, Y.; Yoshida, H.; Kang, H.; Ji, S.; Gopalan, P.; de Pablo, J. J.; 1100 Nealey, P. F. Macromolecules 2013, 46, 1415-1424.

(53) Matsen, M. W. J. Chem. Phys. 1997, 106, 7781. 1102

(54) Drolet, F.; Fredrickson, G. H. Phys. Rev. Lett. 1999, 83, 4317- 1103 4320.

(55) Bosse, A. W.; Garcia-Cervera, C. J.; Fredrickson, G. H. 1105 Macromolecules 2007, 40, 9570.

(56) Hur, S. M.; Garcia-Cervera, C. J.; Kramer, E. J.; Fredrickson, G. 1107 H. Macromolecules 2009, 42, 5861.

(57) Trombly, D. M.; Pryamitsyn, V.; Ganesan, V. J. Chem. Phys. 1109 2011, 134, 154903. 1110

(58) Petera, D.; Muthukumar, M. J. Chem. Phys. 1998, 109, 5101- 1111 5107.

(59) Yang, G.; Tang, P.; Yang, Y.; Cabral, J. T. J. Phys. Chem. B 2009, 1113 $113,14052-14061$. 
1115 (60) Ye, X.; Edwards, B. J.; Khomami, B. Macromolecules 2010, 43, $11169594-9597$.

1117 (61) Kriksin, Y. A.; Neratova, I. V.; Khalatur, P. G.; Khokhlov, A. R. 1118 Chem. Phys. Lett. 2010, 492, 103-108.

1119 (62) Wang, Q.; Nath, S. K.; Graham, M. D.; Nealey, P.; de Pablo, J. J. 1120 J. Chem. Phys. 2003, 118, 11278.

1121 (63) Wang, Q.; Nealey, P. F.; de Pablo, J. J. Macromolecules 2003, 36, 1122 1731-1740.

1123 (64) Detcheverry, F. A.; Pike, D. Q.; Nagpal, U.; Nealey, P. E.; de 1124 Pablo, J. J. Phys. Rev. Lett. 2009, 102, 197801.

1125 (65) Nagpal, U.; Muller, M.; Nealey, P. F.; de Pablo, J. J. ACS Macro 1126 Lett. 2012, 1, 418.

1127 (66) Ohta, T.; Kawasaki, K. Macromolecules 1986, 19, 2621.

1128 (67) Oono, Y.; Puri, S. Phys. Rev. Lett. 1987, 58, 836.

1129 (68) Hamley, I. W. Macromol. Theory Simul. 2000, 9, 363.

1130 (69) Pinna, M.; Zvelindovsky, A. V. Eur. Phys. J. B 2012, 85, 210.

1131 (70) Chakrabarti, A.; Chen, H. J. Polym. Sci., Part B: Polym. Phys.

1132 1998, 36, 3127.

1133 (71) Chen, L. Q.; Shen, J. Comput. Phys. Commun. 1998, 108, 147.

1134 (72) Feng, J.; Liu, H.; Hu, Y. Macromol. Theory Simul. 2002, 11, 549.

1135 (73) Feng, J.; Liu, H.; Hu, Y. Macromol. Theory Simul. 2002, 11, 556.

1136 (74) Li, W.; Qiu, F.; Yang, Y.; Shi, A.-C. Macromolecules 2010, 43,

11371644.

1138 (75) Li, W.; Xie, N.; Qiu, F.; Yang, Y.; Shi, A.-C. J. Chem. Phys. 2011, 1139134,144901

1140 (76) Ohta, T.; Enomoto, Y.; Harden, J. L.; Doi, M. Macromolecules 1141 1993, 26, 4928.

1142 (77) de Groot, S. R; Mazur, P. Phys. Rev. 1954, 94, 218-224.

1143 (78) Fredrickson, G. H.; Helfand, E. J. Chem. Phys. 1987, 87, 6971144705.

1145 (79) Qi, S.; Wang, Z.-G. Phys. Rev. E: Stat. Phys., Plasmas, Fluids, 1146 Relat. Interdiscip. Top. 1997, 55, 1682.

1147 (80) Zvelindovsky, A. V.; Zatovsky, A. V. Il Nuovo Cimento D 1997, $114819,725-745$.

1149 (81) van Vlimmeren, B. A. C.; Maurits, N. M.; Zvelindovsky, A. V.; 1150 Sevink, G. J. A.; Fraaije, J. G. E. M. Macromolecules 1999, 32, 646-656. 1151 (82) Knoll, A.; Lyakhova, K. S.; Horvat, A.; Krausch, G.; Sevink, G. J. 1152 A.; Zvelindovsky, A. V.; Magerle, R. Nat. Mater. 2004, 3, 886-890.

1153 (83) Horvat, A.; Sevink, G. J. A.; Zvelindovsky, A. V.; Krekhov, A.; 1154 Tsarkova, L. ACS Nano 2008, 2, 1143-1152.

1155 (84) Bahiana, M.; Oono, Y. Phys. Rev. A: At., Mol., Opt. Phys. 1990, $115641,6763$.

1157 (85) Leibler, L. Macromolecules 1980, 13, 1602.

1158 (86) Ren, S. R.; Hamley, I. W. Macromolecules 2001, 34, 116.

1159 (87) Marko, J. F. Phys. Rev. E: Stat. Phys., Plasmas, Fluids, Relat. 1160 Interdiscip. Top. 1993, 48, 2861.

1161 (88) Feng, J.; Ruckenstein, E. J. Chem. Phys. 2004, 121, 1609.

1162 (89) Ren, S. R.; Hamley, I. W.; Sevink, G. J. A.; Zvelindovsky, A. V.; 1163 Fraaije, J. G. E. M. Macromol. Theory Simul. 2002, 11, 123.

1164 (90) Shinozaki, A.; Oono, Y. Phys. Rev. E: Stat. Phys., Plasmas, Fluids, 1165 Relat. Interdiscip. Top. 1993, 48, 2622.

1166 (91) Ren, S. R.; Hamley, I. W.; Teixeira, P. I. C.; Olmsted, P. D. Phys. 1167 Rev. E: Stat. Phys., Plasmas, Fluids, Relat. Interdiscip. Top. 2001, 63, 1168041503.

1169 (92) Pinna, M.; Zvelindovsky, A. V.; Todd, S.; Goldbeck-Wood, G. J. 1170 Chem. Phys. 2006, 125, 154905.

1171 (93) Pinna, M.; Zvelindovsky, A. V.; Guo, X.; Stokes, C. L. Soft 1172 Matter 2011, 7, 6991.

1173 (94) Vega, D. A.; Harrison, C. K.; Angelescu, D. E.; Trawick, M. L.; 1174 Huse, D. A.; Chaikin, P. M.; Register, R. A. Phys. Rev. E 2005, 71, 1175061803.

1176 (95) Komura, S.; Fukuda, J.-I.; Paquette, G. C. Phys. Rev. E: Stat.

1177 Phys., Plasmas, Fluids, Relat. Interdiscip. Top. 1996, 53, R5588.

1178 (96) Brazovskii, S. A. Sov. Phys. JETP 1975, 41, 85.

1179 (97) Stein, G. E.; Cochran, E. W.; Katsov, K.; Fredrickson, G. H.; 1180 Kramer, E. J.; Li, X.; Wang, J. Phys. Rev. Lett. 2007, 98, 158302. 